\title{
Le monument funéraire néolithique de Poses « Sur la Mare » (Eure)
}

Cyrille Billard, Isabelle Le Goff, Jacques Évin, Jan Lanting, Françoise Reckinger et André-Valentin Munaut

\section{(2) OpenEdition \\ 12 Journals}

Édition électronique

URL : https://journals.openedition.org/rao/60

DOI : $10.4000 /$ rao. 60

ISBN : 978-2-7535-1606-9

ISSN : 1775-3732

Éditeur

Presses universitaires de Rennes

Édition imprimée

Date de publication : 30 décembre 2006

Pagination : 87-115

ISBN : 978-2-7535-0574-2

ISSN : 0767-709X

\section{Référence électronique}

Cyrille Billard, Isabelle Le Goff, Jacques Évin, Jan Lanting, Françoise Reckinger et André-Valentin Munaut, "Le monument funéraire néolithique de Poses « Sur la Mare » (Eure) », Revue archéologique de I'Ouest [En ligne], 23 | 2006, mis en ligne le 30 décembre 2008, consulté le 18 août 2022. URL : http:// journals.openedition.org/rao/60 ; DOI : https://doi.org/10.4000/rao.60 


\title{
Le monument funéraire néolithique de Poses «Sur la Mare» (Eure)
}

\author{
Cyrille Billard* et Isabelle Le GofF* \\ avec la collaboration de Jacques Évin***, Jan Lanting ${ }^{* * * *}$, \\ Françoise ReCKInGER ${ }^{* * * * *}$ et André-Valentin Munaut ${ }^{* * * * *}$
}

\begin{abstract}
Résumé : La fouille d'une large surface, à Poses, au lieu-dit "Sur la Mare ", a permis d'étudier les vestiges d'une petite structure funéraire en partie arasée. Cet édifice est constitué d'une couronne d'empierrement en blocs de craie, de forme ovalaire et délimitant un espace central quadrangulaire d'environ $4 \mathrm{~m}$ sur 6 . Cet espace intérieur, légèrement excavé, a fourni d'une part une structure contenant un vase, et d'autre part une nappe d'ossements brûlés associés à d'autres restes également brûlés : fragments de céramique, outillages osseux et lithique. Les questions concernant le type de fonctionnement, l'architecture et la datation de cet édifice à une phase de transition entre les Néolithiques moyen et récent font ici l'objet d'une discussion.
\end{abstract}

\begin{abstract}
The neolithic burial structure of Poses "Sur la Mare" (Eure)
Area excavation at Poses "Sur la Mare" revealed a small Neolithic burial structure. This partly eroded monument consisted of an oval surround of chalk blocks, defining a central quadrangular area 4 by 6 metres. This space, slightly hollowed, had in one section a feature containing a pot, in another section a deposit of cremated bones associated with other burnt material: shards, worked bone and fints. Discussion centres on the function, the architecture and the dating of this structure in a transitional phase between the middle and late Neolithic.
\end{abstract}

Mots clés : Monument, funéraire, incinération, néolithique.

Key words: Monument, funerary, cremation, Neolithic.

\section{INTRODUCTION}

En 1995, une fouille importante a été réalisée sur une superficie d'environ 4,5 hectares au lieu-dit "Sur la Mare » à Poses (Eure), à l'emplacement de la carrière d'extraction de granulats de l'entreprise Morillon-Corvol. Les vestiges étudiés concernaient un village du Néolithique ancien (groupe de Villeneuve-Saint-Germain), une structure funéraire néolithique, un habitat fossoyé protohistorique associé à des maisons circulaires et enfin un habitat de l'époque galloromaine, cf. Bostyn (dir.), 2003 (fig. 13, p. 32).

Limportance et la diversité des vestiges avaient imposé de partager le site en deux interventions distinctes, l'une pour la partie Néolithique ancien (cf. Bostyn [dir.], 2003) et l'autre pour les vestiges protohistoriques et gallo-romains (cf. Langlois et Celly, 1995). De plus, un enclos trapézoïdal de l'âge du Fer a été fouillé par T. Dechezleprêtre, tandis que ce qui s'apparentait a priori à une structure tumulaire

* DRAC de Basse-Normandie, Service régional de l'Archéologie, 13bis rue Saint-Ouen, 14052 Caen cedex 04.

** INRAP Centre - Ille-de-France - UMR 7041, 40 place G.-Lyssandre, 93140 Bondy.

*** Université de Lyon 1, Centre de Datations par le Radiocarbone, 40 boulevard Niels-Bohr, 69622 Villeurbanne cedex.

**** Université de Groningen (Pays-Bas), Institut voor Archeologie, Poststraat 6, 9712 ER Groningen.

***** Université catholique de Louvain (Belgique), Laboratoire de Palynologie et de Dendrochronologie, Place Croix-du-sud, 5, Bte 11, B-1348 Louvainla-Neuve. 
néolithique ou de l'âge du Bronze a été pris en charge par l'un d'entre nous (C. Billard), tous ayant travaillé en totale concertation. Seules les structures funéraires appartenant au Néolithique sont présentées ici.

\section{Présentation générale}

\section{Le Contexte}

La structure funéraire de Poses est situé dans la Boucle du Vaudreuil, à la confluence de la Seine et de l'Eure. La plaine alluviale atteint ici une largeur exceptionnelle, proche de $5 \mathrm{~km}$, renforcée par la confluence de l'Andelle au nord de la boucle au niveau de Pîtres (fig. 1). Cette entité géographique forte est marquée par le passage de la Seine à l'est et au nord, de l'Eure à l'ouest. La Boucle du Vaudreuil offre une grande diversité de milieux, ce qui a dû constituer un certain attrait pour les populations néolithiques : la plaine est dominée par les plateaux à couverture limoneuse et les coteaux calcaires. En rive convexe du méandre, une succession de gradins à pente douce correspond aux terrasses alluviales anciennes de la Seine.

La partie centrale de la boucle est formée essentiellement par la très basse terrasse, recouverte d'alluvions grossières et qui constitue une plate-forme d'altitude comprise entre +7 et $+12 \mathrm{~m}$ NGF. Les zones basses de ce secteur sont particulièrement développées au nord et au sud, où elles ont été colmatées par des alluvions fines holocènes et fréquemment soumises aux inondations périodiques de la Seine. La nappe alluviale constituant le substrat du site étudié est formée essentiellement de sables et de galets de silex. Elle comporte quelques blocs erratiques de faibles dimensions (rarement plus longs que $1,50 \mathrm{~m}$ ), en grès ou en meulière et très exceptionnellement en calcaire. La craie ne se trouve, au plus près, que dans les falaises situées sur les rives opposées de la Seine et de l'Eure, à plus de $2 \mathrm{~km}$ à vol d'oiseau, sous la forme de craies blanches à Belemnitelles (C8, Sénonien supérieur) et à Micraster (C7, Sénonien moyen et inférieur).

La plaine alluviale de la confluence Seine-Eure est une zone riche en sépultures collectives avec un total de neuf sites dénombrés (fig. 1; Billard et al., 1995). Les monuments de Saint-Étienne-du-Vauvray, de Léry et de Pinterville (au sud d'Incarville) ont fait l'objet de fouilles anciennes (1842, 1874 et 1943), tandis que l'ensemble des sépultures de Val-de-Reuil et Portejoie - cinq allées sépulcrales en cours de publication - est localisé au sud-est de la Boucle du Vaudreuil sur une partie de cette très basse terrasse (Verron, 1975; Billard et al., en préparation).

- La sépulture de la Basse-Crémonville à Saint-Étiennedu-Vauvray est décrite comme une fosse circulaire de 4,50 m de diamètre recouverte par une dalle unique. La description de la position des squelettes est peu crédible, mais il semble que, d'après les fouilleurs, la stratigraphie ait comporté trois couches d'ossements séparées entre elles par des dallages; le mobilier est constitué de gaines en bois de cerf à emmanchement transversal, caractéristiques du complexe funéraire SOM. Un deuxième monument, qui n'a pu être fouillé, a semble-t-il été repéré au même moment à environ un kilomètre du précédent en direction de Louviers (Bonnin, 1843; Collignon, 1928-1930).

- Le dolmen des Vignettes à Léry est un petit caveau mégalithique en fer à cheval, partiellement enseveli dans des alluvions récentes provenant du débordement de l'Eure et de la Seine. Il était composé de huit dalles verticales, d'une dalle allongée en guise de fermeture et d'une dalle de couverture de 2,70 x 1,40 m (Hamy, 1874; Coutil, 1896). La description par Coutil du mobilier découvert est assez imprécise : « deux hachettes perforées en jadéite, trois haches polies en silex, plusieurs couteaux en silex, des colliers et pièces d'enfilage naturelles, deux amulettes (dont l'une sur fragment de bracelet de schiste), une pointe de flèche, un peigne en os, plusieurs gaines à emmanchement transversal, des poinçons en os, plusieurs petits vases en terre grise grossière et rougeâtre, des débris de ruminant ». Deux petits vases sont conservés, l'un au musée de Louviers, l'autre au musée d'Évreux.

- Le monument de Pinterville est une allée sépulcrale de $10 \mathrm{~m}$ de long ayant contenu environ cinquante individus (Baudot et Gaudron, 1943; Baudot, 1944; Divry, 1944; Marquer, 1954). Découverte en 1942 lors du creusement d'une tranchée par les troupes allemandes, elle est orientée nord-sud avec son entrée au sud, le vestibule étant séparé de la chambre funéraire par une dalle échancrée. Les corps, placés au niveau du pavage de la chambre, présentaient des ossements en connexion anatomique; la disposition primitive des corps semble avoir été «l'allongement dans le sens de la longueur de l'allée, les pieds au nord, la tête au sud, et probablement couchés sur le dos ». Outre deux vases de l'âge du Bronze ou du début de l'âge du Fer découverts au sommet et à l'entrée du caveau, ce monument a livré un riche mobilier néolithique : "Pointe de javelot en silex taillé (?), fragment de palette en céramique (?), cinq poinçons en os poli, une hache polie en roche verte, une pierre verte polie, une défense de suidé biforée, une dent de renard perforée, cinq grosses pierres percées, un gros os long percé, un os de gros ruminant percé, un cylindre percé en ivoire, deux grosses perles translucides, une perle en céramique rougeâtre, quatre coquillages fossiles percés, deux os percés à ailettes, quinze perles en os en barillet, 85 petites perles plates en os, 168 petites perles plates en nacre et 257 petites perles plates en jayet. " Parmi le mobilier qui a été déposé à l'Institut de Paléontologie humaine après la fouille, nous n'avons pu 

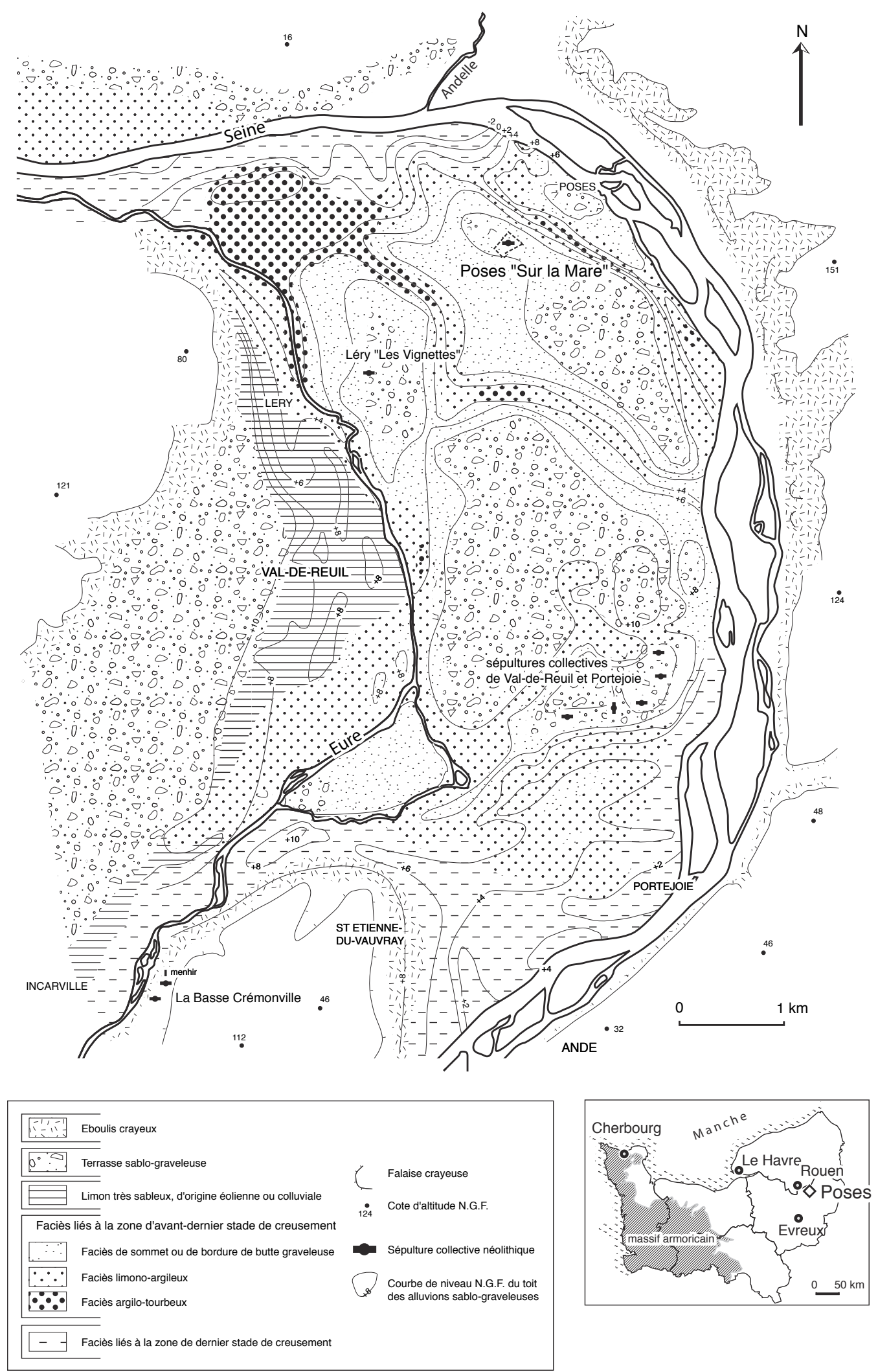

Figure 1 : Poses « Sur la Mare »; localisation, contexte géomorphologique et monuments néolithiques voisins du site.

Figure 1: Location, geomorphological context and local neolithic monuments. 
retrouver que onze éléments de parure, un des deux récipients protohistoriques ainsi que des fragments céramiques appartenant à trois vases du Néolithique final, à fond plat et profil en $S$.

La Boucle du Vaudreuil a également livré plusieurs sites d'habitat, jusqu'à présent jamais situés dans l'environnement immédiat de ces monuments.

\section{Stratigraphie générale du site}

La structure funéraire de "Sur la Mare » a été repérée dès les premiers sondages en 1994 sous la forme d'un empierrement arqué (fig. 2). Après décapage complet du sommet de l'empierrement (fig. 3), celui-ci a été dégagé sur quelques mètres carrés seulement et un sondage manuel a été réalisé dans l'espace central délimité partiellement par les couches de pierres (fig. 4). Le hasard a fait que ce sondage était localisé dans une zone sans structure remarquable et quasiment sans mobilier. Il a néanmoins montré que, d'une part, la partie centrale était nettement excavée et que, d'autre part, les quelques tessons épars recueillis étaient plutôt de facture néolithique (confirmant que la structure n'était pas une fondation de bâtiment gallo-romain, comme on l'avait imaginé au départ). Un premier fragment d'outil en os calciné a également été trouvé dans ce sondage.

D'une manière simplifiée, l'épaisseur de sédiment audessus de l'empierrement est importante, avec en surface environ $40 \mathrm{~cm}$ de terre végétale et environ 20 à $30 \mathrm{~cm}$ de limons de débordement légèrement argileux. Le dépôt de ces limons est sans aucun doute récent puisqu'un tesson du haut Moyen Âge décoré à la molette et quelques autres petits tessons médiévaux ont été trouvés au sommet de l'empierrement.

Comme nous le verrons, l'empierrement semble avoir été aménagé directement sur le sol néolithique dont le sommet se trouve généralement 10 ou $20 \mathrm{~cm}$ au-dessus du niveau de la grave. La conservation d'une partie de son élévation est donc due au contexte très particulier d'exposition du site aux crues de la Seine et au dépôt important de limons de débordement.

La zone de paléosol conservée correspond globalement à l'espace empierré mais également à la partie occidentale de la zone centrale excavée. Autour de la zone empierrée, s'observe une vaste dépression encaissée d'environ 10 à $20 \mathrm{~cm}$ dans le substrat sablo-graveleux (fig. 2). Cette dépression est colmatée par un limon sableux dans lequel ont été trouvés des vestiges mobiliers, avec en particulier deux petites concentrations de tessons. L'hypothèse la plus sérieuse est que les sédiments environnant la structure funéraire ont été raclés pour servir à réaliser une masse tumulaire.
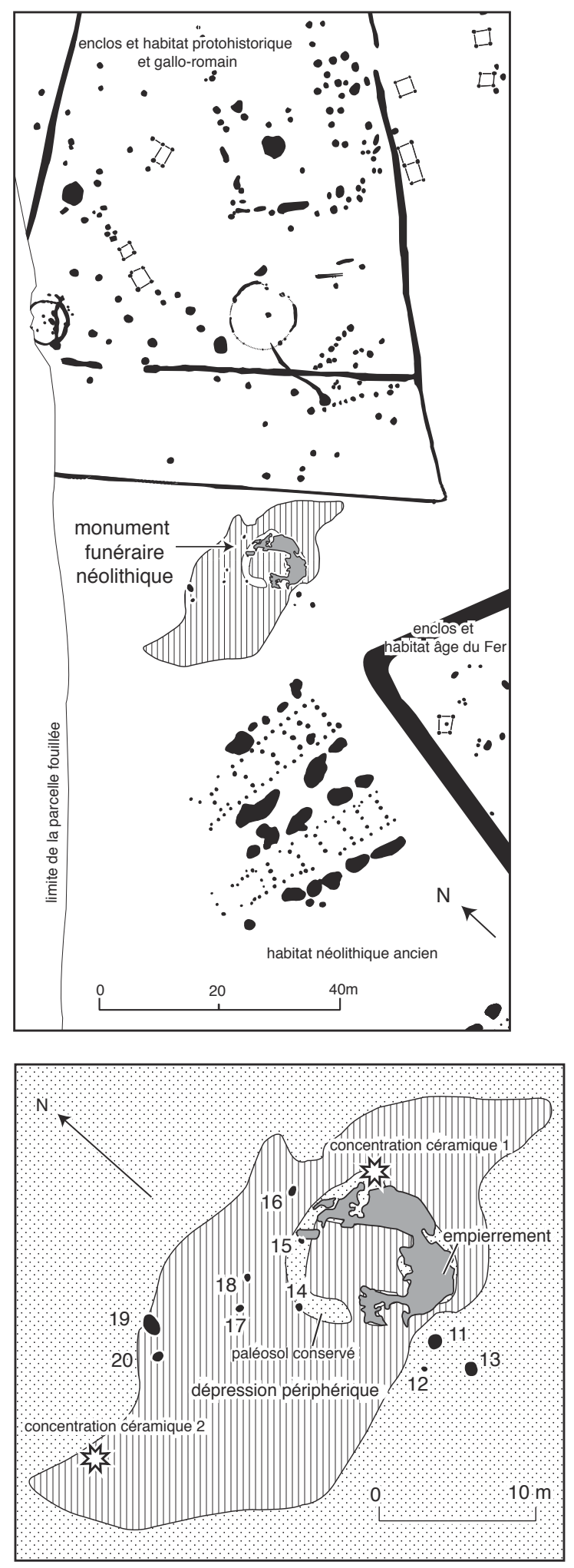

Figure 2 : Poses "Sur la Mare», environnement archéologique proche ; en bas, plan de l'édifice, de sa dépression périphérique et de ses structures proches.

Figure 2: Local archaeological environment; below, plan of the structure, its surrounding hollow and adjoining features. 


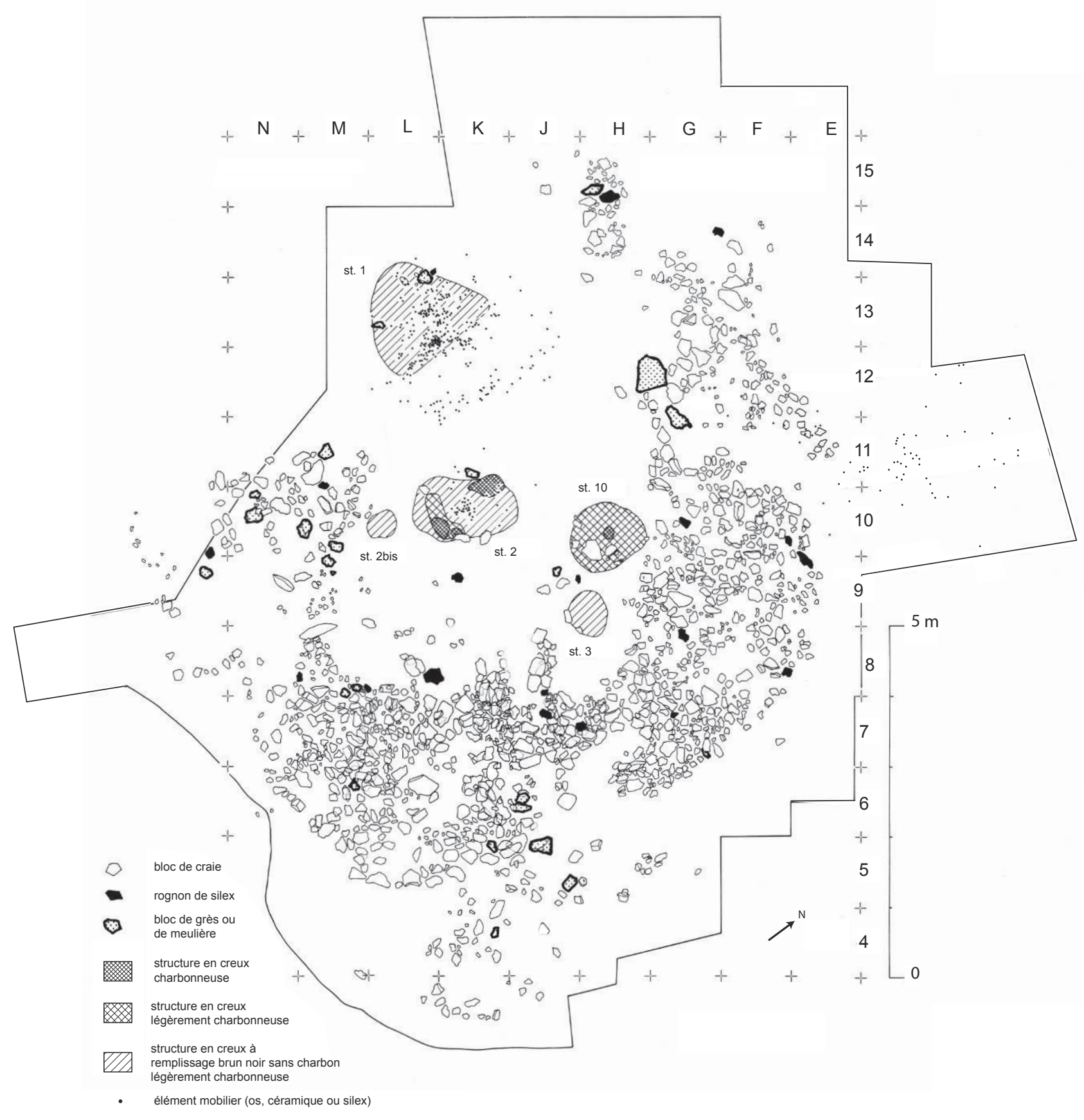

Figure 3 : Plan détaillé des vestiges d'empierrement, des structures internes et répartition générale du mobilier.

Figure 3: Detailed plan of the surround, the internal structures and general distribution of finds.

\section{Méthodes de fouille}

Après le décapage mécanique complet qui a permis la mise au jour du sommet de l'empierrement, un carroyage métrique orienté par rapport au carroyage général du site a été mis en place (fig. 3). Une fois le sondage manuel réalisé, le sommet de l'empierrement a été dégagé, puis ont été réalisés deux transects orthogonaux au moyen de deux tranchées (coupes 1 et 2, fig. 4). À ce stade, des taches de sédiment brun noir ainsi que quelques esquilles d'os brûlés (en KL13) sont apparues en surface du décapage de l'espace central non empierré. Une fouille fine a alors été menée sur l'ensemble de la partie délimitée par l'empierrement, en ménageant des banquettes témoins. L’ensemble des vestiges mobiliers a été 


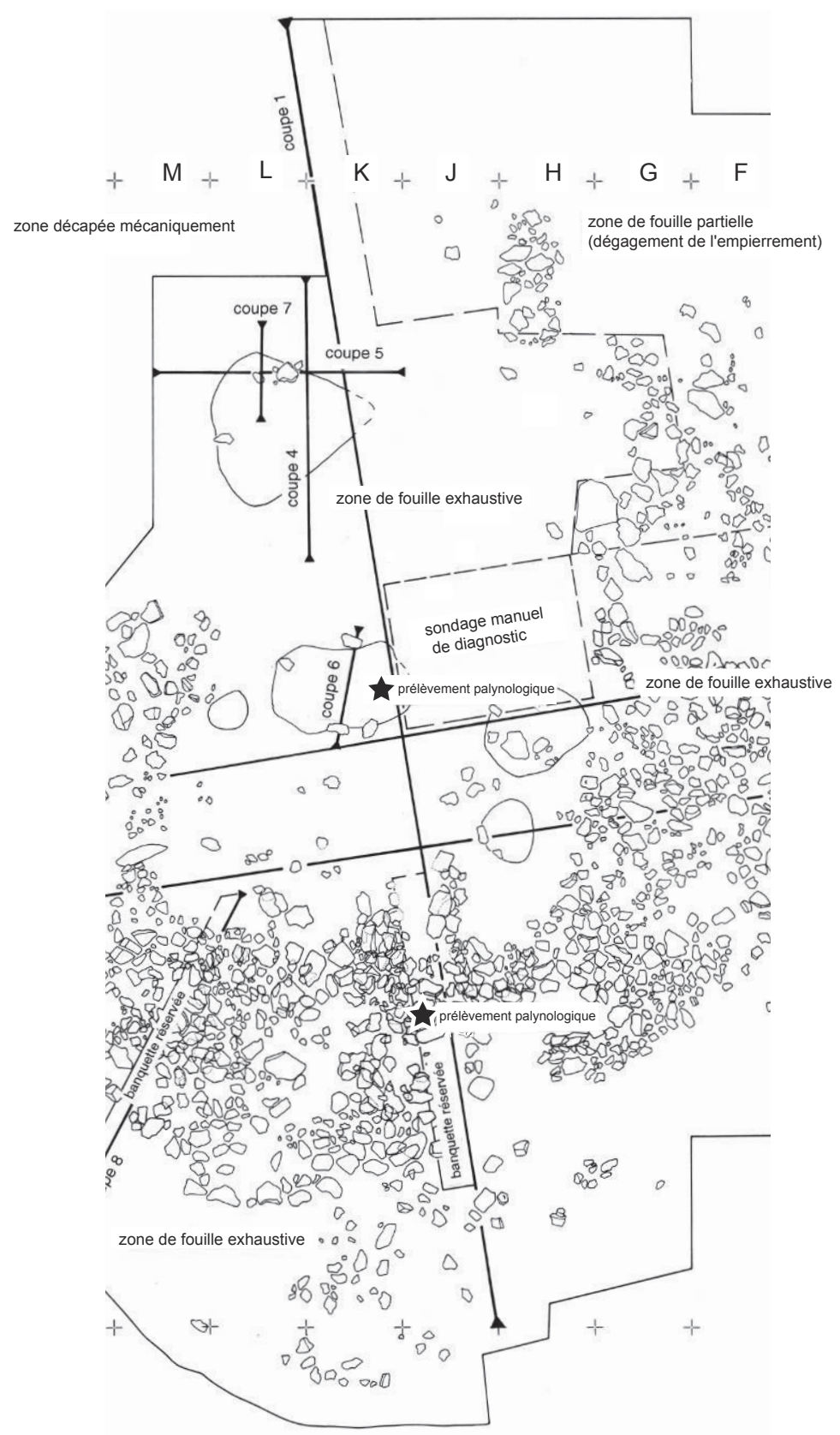

Figure 4 : Localisation des coupes et des prélèvements palynologiques.

Figure 4: Location of sections and pollen samples.

dessiné et coté en altitude. Les petits galets brûlés ont été prélevés par mètre carré et par structure.

Des numéros ont été attribués à l'ensemble des structures observées, y compris les bioturbations (terriers) (fig. 5). Ces dernières ont largement perturbé la structure funéraire, mais leur identification en tant que telles n'a eu lieu qu'à une phase avancée de la fouille. Le doute quant à leur nature exacte a pu être en grande partie levé lorsque l'ensemble des colluvions environnant le monument a été décapé mécaniquement : à ce moment, sont apparus d'autres réseaux de fosses formant des circonvolutions anarchiques nous encourageant dans cette hypothèse. Très probablement liée à la présence d'animaux fouisseurs sous le monument, leur nature exacte reste hypothétique dans la mesure où aucun vestige, tel que des ossements animaux, n'en atteste la présence.

Pour ce qui concerne la structure funéraire, la fouille de l'empierrement et de ses parties internes et externes a livré du matériel remanié et souvent très émoussé. L'enregistrement de ce matériel épars a été simplifié au maximum en zones 


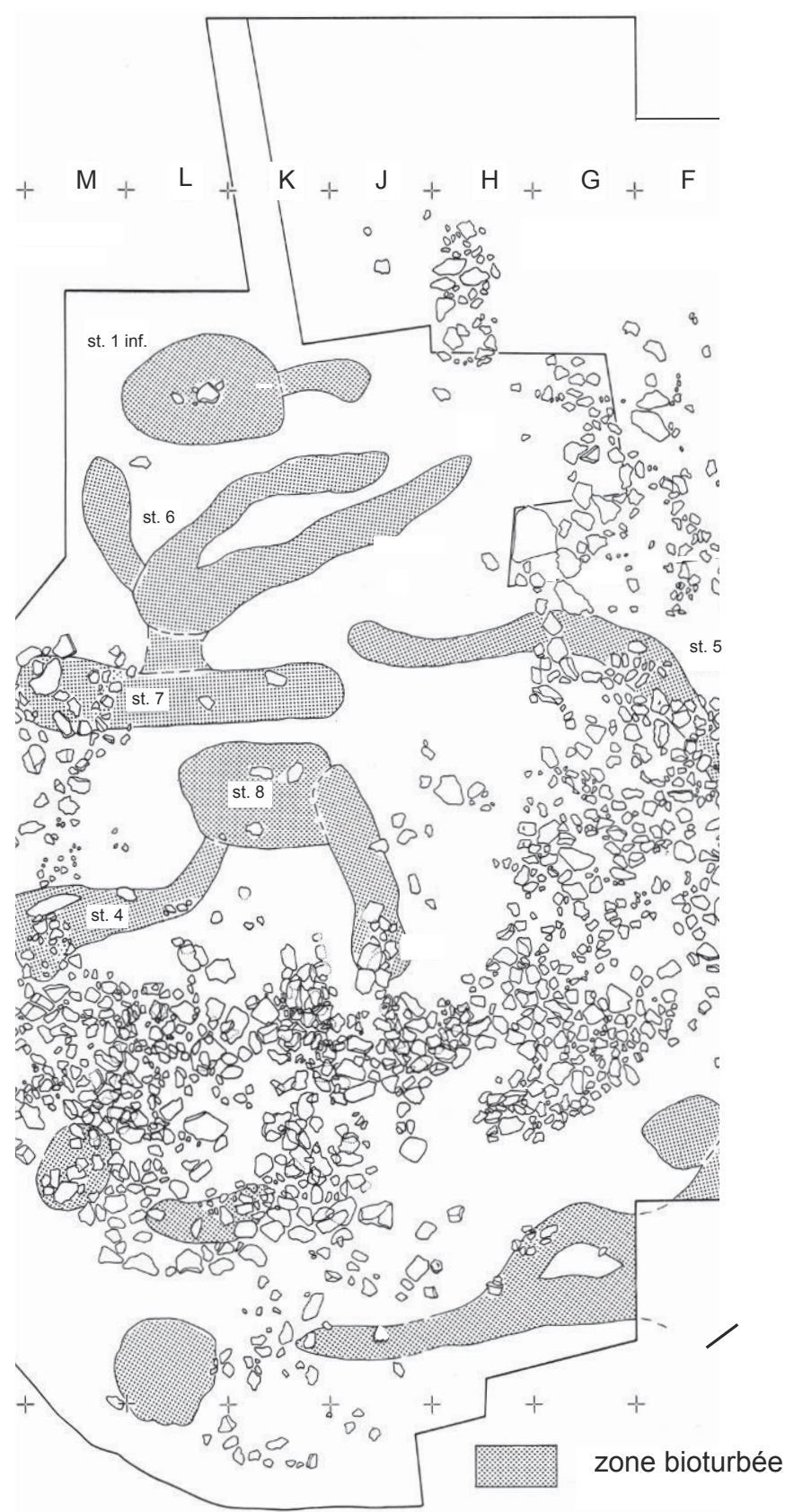

Figure 5 : Répartition des zones probablement bioturbées.

Figure 5: Location of areas probably disturbed.

numérotées de 1 à 11 , qui ont été localisées en fonction de l'emplacement des coupes (et dont on pourra trouver le plan dans le rapport de fouille déposé au SRA de BasseNormandie). Ces zones étant choisies arbitrairement, nous avons indiqué, lorsque cela était nécessaire, si le mobilier provenait de l'empierrement lui-même, de la partie interne de la structure ou bien de la partie externe (dépression de limons sableux). À la fin de la fouille manuelle du monument, l'ensemble des zones non fouillées (pour l'essentiel dans la dépression périphérique) a fait l'objet d'un deuxième décapage mécanique jusqu'au sommet de la grave. Des numéros de structures (jusqu'à la structure 20) ont été également attribués à des fosses identifiées lors de cette dernière phase. 


\section{LES STRUCTURES ARCHÉOLOGIQUES}

\section{La zone empierrée et sa dépression périphérique}

Autour de la zone empierrée s'étend une vaste zone dépressionnaire, encaissée de 10 à $20 \mathrm{~cm}$ dans la grave et colmatée de limons sableux. Cette dépression recouvre une surface d'environ $414 \mathrm{~m}^{2}$ (hors monument), de forme losangique, très étirée vers l'ouest et l'est (fig. 2). Dans toutes les zones de la fouille, figuraient des vestiges épars correspondant à du mobilier remanié lors d'apport de matériaux provenant des environs du site : produits de débitage en silex, tessons émoussés d'époques et de technologies diverses (Villeneuve-saint-Germain, Néolithique récent/final), ossements de faune très fragmentés et très érodés. Un tesson décoré d'incisions en arêtes et une anse en boudin signalent très probablement le prélèvement de sédiments dans l'environnement des maisons du groupe de Villeneuve-saintGermain avoisinantes (la fosse VSG la plus proche étant située à environ $15 \mathrm{~m}$ au sud-ouest de l'empierrement).

Dans cette dépression, l'empierrement couvre une surface de forme ovale de 8,8 x 11,2 m, orientée nord-ouest - sudest, à laquelle il faut retrancher la zone centrale et de probables zones de prélèvements de blocs, principalement dans sa partie ouest (fig. 3). La régularité de sa limite externe orientale peut laisser présager de l'existence d'un parement périphérique. Il est constitué pour l'essentiel de petits blocs de craie, dont les plus grands ne dépassent pas $40 \mathrm{~cm}$ de longueur, et qui présentent des traces de décalcification leur donnant un aspect très émoussé. Très marginalement, figurent également des rognons bruts de silex, des blocs de grès ou de meulière dont certains sont parfois brûlés. Une meule de grès y est également en réemploi.

L'ensemble de l'empierrement repose le plus souvent sur une couche de sable limoneux brun clair directement susjacente au substrat sablo-graveleux et que l'on peut qualifier de paléosol (fig. 6 et 7). Cet empierrement est relativement bien conservé dans sa partie sud-est, où il est constitué de deux, voire trois niveaux de blocs superposés. Par contre, au nord-est et au sud-ouest, il n'y a plus qu'un seul niveau de blocs, tandis qu'à l'ouest ils sont absents. Ceux-ci ont pu avoir été prélevés pour être réemployés ailleurs, comme le suggèrent plusieurs alvéoles vides de vestiges et situées le long de la limite externe (en N8-9 et en HJ-6-7 ; cf. fig. 2 et 3). Ces zones sans blocs n'ont généralement pas été affectées par le creusement de la dépression périphérique. La conservation du paléosol à cet emplacement est un argument permettant de penser que l'empierrement encerclait à l'origine la zone centrale des vestiges osseux et céramiques. Quelques très rares blocs de petit module se retrouvent également en périphérie immédiate de l'empierrement.
L'origine des blocs de craie et des rognons de silex est relativement éloignée du site, puisqu'ils ne sont accessibles qu'en traversant la Seine ou l'Eure sur les coteaux environnants, soit à une distance supérieure à $2 \mathrm{~km}$. Les autres matériaux sont locaux.

Le mobilier recueilli au niveau de l'empierrement comporte également, en faible quantité, des fragments de faune, des produits de débitage du silex et des tessons de technologies diverses (présence de tessons VSG), qui semblent tous largement remaniés.

\section{La partie centrale de la zone empierrée}

La zone centrale délimitée par l'empierrement forme de manière schématique un rectangle de 4 x $6 \mathrm{~m}$ dont la surface est notablement creusée par rapport au substrat, à l'exception d'une petite surface d'environ $1 \mathrm{~m}^{2}$ située le long du bord sud-est de l'empierrement (fig. 3 et 6 à 9). Cette partie non creusée correspond d'ailleurs à une légère extension des blocs à l'intérieur du rectangle. Nous pouvons considérer que les limites de la partie ouest, moins perceptibles du fait de l'absence d'empierrement, correspondent à la limite de la zone excavée.

Le sédiment de remplissage de cette partie centrale est comparable au sédiment encaissant de l'empierrement (limon très sableux brun), avec cependant une couleur légèrement plus foncée. On y trouve également de façon éparse du mobilier remanié (silex, tesson, faune). Les aménagements et dépôts funéraires qui ont pu y être observés apparaissent généralement à la surface du limon sableux ou à une altitude légèrement inférieure. Ils sont le plus souvent très perturbés par la présence de terriers d'animaux fouisseurs sur desquels nous reviendrons.

\section{La «structure 1 » et son mobilier}

\section{Description}

Il s'agit d'une nappe de sédiment brun noir, sans charbon apparent, qui s'étend sur plus de $2 \mathrm{~m}^{2}$ avec des contours assez flous. La répartition des vestiges associés déborde largement la limite de ce sédiment, probablement à cause des bioturbations. Cette structure occupe l'angle occidental de la surface rectangulaire délimitée en partie par l'empierrement. En altitude, cette nappe se situe environ $10 \mathrm{~cm}$ plus bas que la base de l'empierrement. Le caractère marquant de cette poche est qu'elle appartient à un épisode tardif du colmatage limono-sableux de la partie centrale. Les autres vestiges mobiliers découverts à des niveaux plus profonds s'inscrivent nettement dans les limites des terriers et sont donc remaniés.

Les vestiges y sont tous brûlés. Ils comportent près de 300 esquilles d'ossements humains et, dans une moindre mesure, 


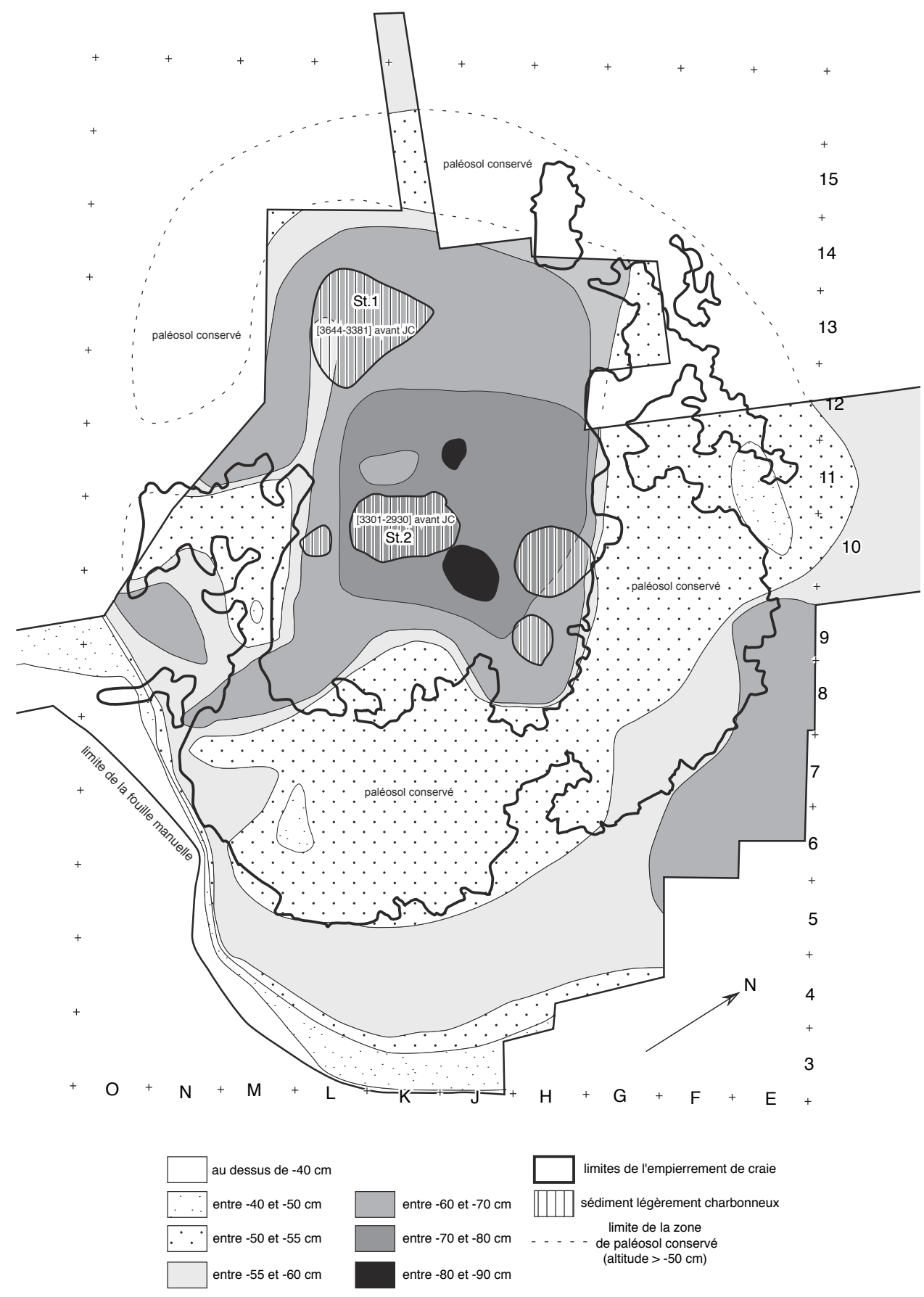

Figure 6 : Courbes de niveau du sommet de la grave (restituées sans tenir compte des perturbations engendrées par les animaux fouisseurs).

Figure 6: Contours of the gravel summit (restored not taking in account disturbance by animal burrowing).

animaux, 79 tessons appartenant pour l'essentiel à un récipient unique (en dehors de quelques autres tessons isolés provenant probablement du sédiment rapporté), 16 fragments d'outils en os, 1 ciseau en silex et 1 armature de flèche tranchante brûlée. À l'emplacement de la nappe brun noir, on trouve des galets brûlés (module compris entre 1 et $8 \mathrm{~cm}$ ), dont le poids total atteint environ $2 \mathrm{~kg}$, et quelques autres non brûlés.

\section{La céramique}

Hormis les rares tessons isolés qui ne semblent pas en place et qui ne sont pas spatialement associés au reste des vestiges brûlés, les 79 tessons numérotés dans la structure 1 et à proximité appartiennent à un récipient qui a très probablement subi une double cuisson (A, fig. 10). L'unicité du vase est très probable, mais pas totalement assurée car les tessons 

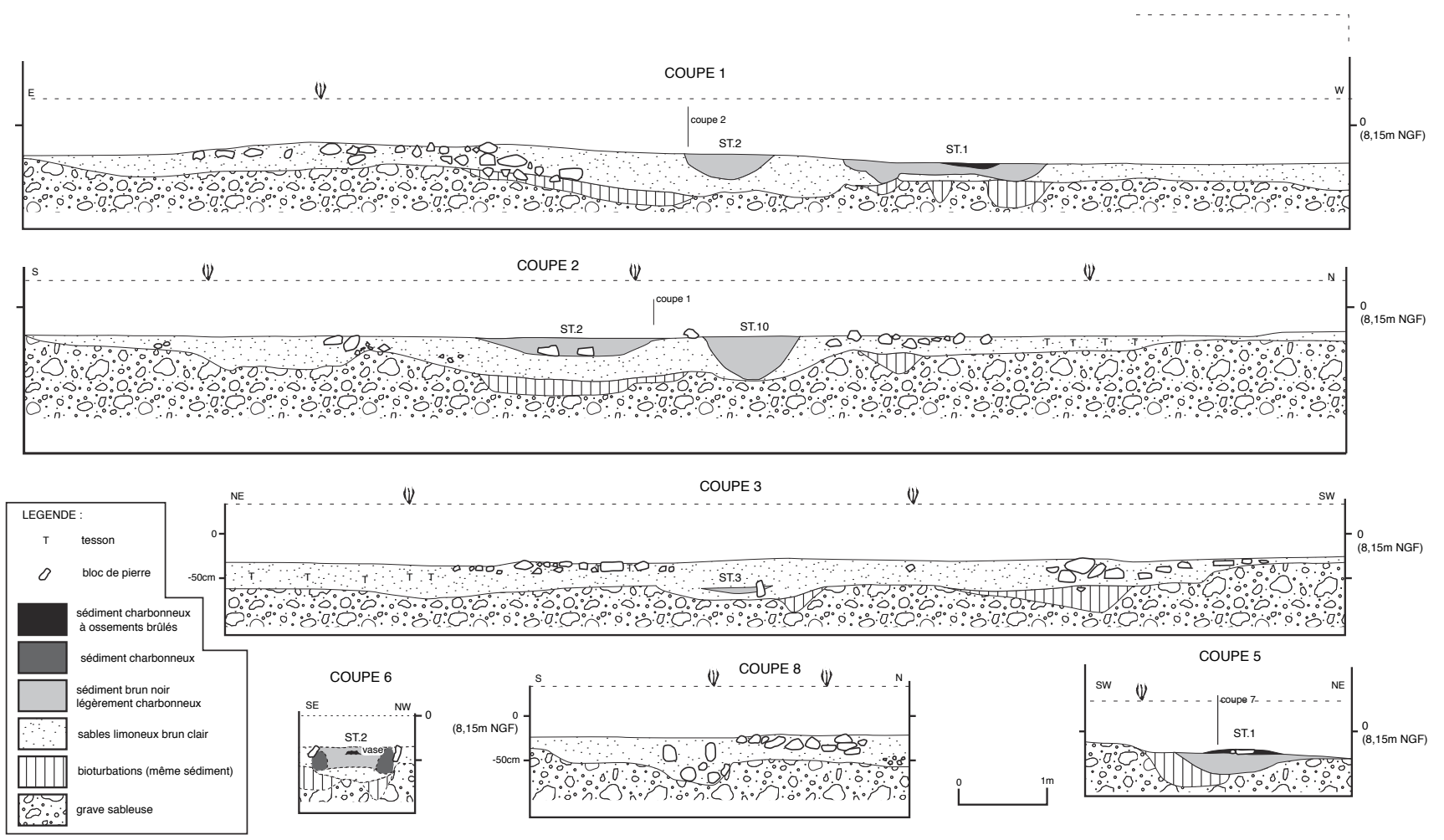

Figure 7 : Coupes stratigraphiques.

Figure 7: Stratigraphy.

sont largement déformés, soit aplatis, soit quasiment pliés; de plus, les remontages sont rendus difficiles par l'émiettement des tranches, si bien qu'il est extrêmement délicat de proposer une restitution complète de sa forme.

Un réseau de larges fissures couvre l'ensemble de la surface du vase et, par endroits, la pâte est presque vitrifiée avec formation de « micro bulles». La dimension des tessons est variable, mais les plus gros atteignent $10 \mathrm{~cm}$. Leur couleur est globalement brun orangé à brun noir et ils possèdent un dégraissant composé de morceaux de silex et de chamotte. L'épaisseur moyenne de la pâte est de $1,2 \mathrm{~cm}$. Le fond du vase est plat et non débordant, son bord est aminci, mais les certitudes s'arrêtent là. Le mauvais état de la céramique explique que seuls sept remontages aient été réalisés. Cependant, on peut affirmer qu'il s'agit d'une forme simple à fond plat et à parois légèrement galbées, présentant un bord subvertical et d'un diamètre inférieur à $20 \mathrm{~cm}$.

L'action du feu sur le récipient est indiscutable et trouve des comparaisons, par exemple au travers des déformations subies par les urnes cinéraires de la nécropole gauloise d'Epiais-Rhus (Val-d'Oise) (Maire, 1983). Sur ce site, un même récipient peut présenter un côté non affecté, de simples fissures ou bien un changement général de la couleur des parois. Le stade le plus avancé de l'exposition au feu aboutit à des vases affaissés à pâte mâcheferisée et bulbeuse.
L'industrie en silex

Deux objets peuvent être associés de manière quasi certaine au reste du mobilier osseux et funéraire $\left(\mathrm{n}^{\circ} 12\right.$ et 13 , fig. 11). Il s'agit d'une armature de flèche à tranchant transversal et d'un outil pouvant évoquer un tranchet étroit. Les deux objets étaient distants l'un de l'autre de $30 \mathrm{~cm}$ et appartiennent à la plus forte concentration de mobilier observée dans cette structure, dans le quart est du carré L13. Ils portent chacun les traces d'une cuisson subie dans des conditions de température identiques : le silex est devenu gris et porte de petites craquelures, avec parfois de petits éclatements. Il manque une partie de la base de l'armature.

Ces deux objets ont pu être introduits dans le monument avec les terres prélevées aux environs, mais cette hypothèse résiste mal aux données archéologiques. En effet, ils représentent les seuls éléments lithiques façonnés de la structure et sont les seuls à avoir subi les effets d'une combustion. De plus, ils sont localisés au sein de la plus forte concentration de mobilier osseux et céramique. Enfin, Ils se rattachent chronologiquement au Néolithique moyen-final, période qui n'est pas représentée dans les environs du monument par d'autres structures. Pour toutes ces raisons, leur association semble quasi-certaine. 


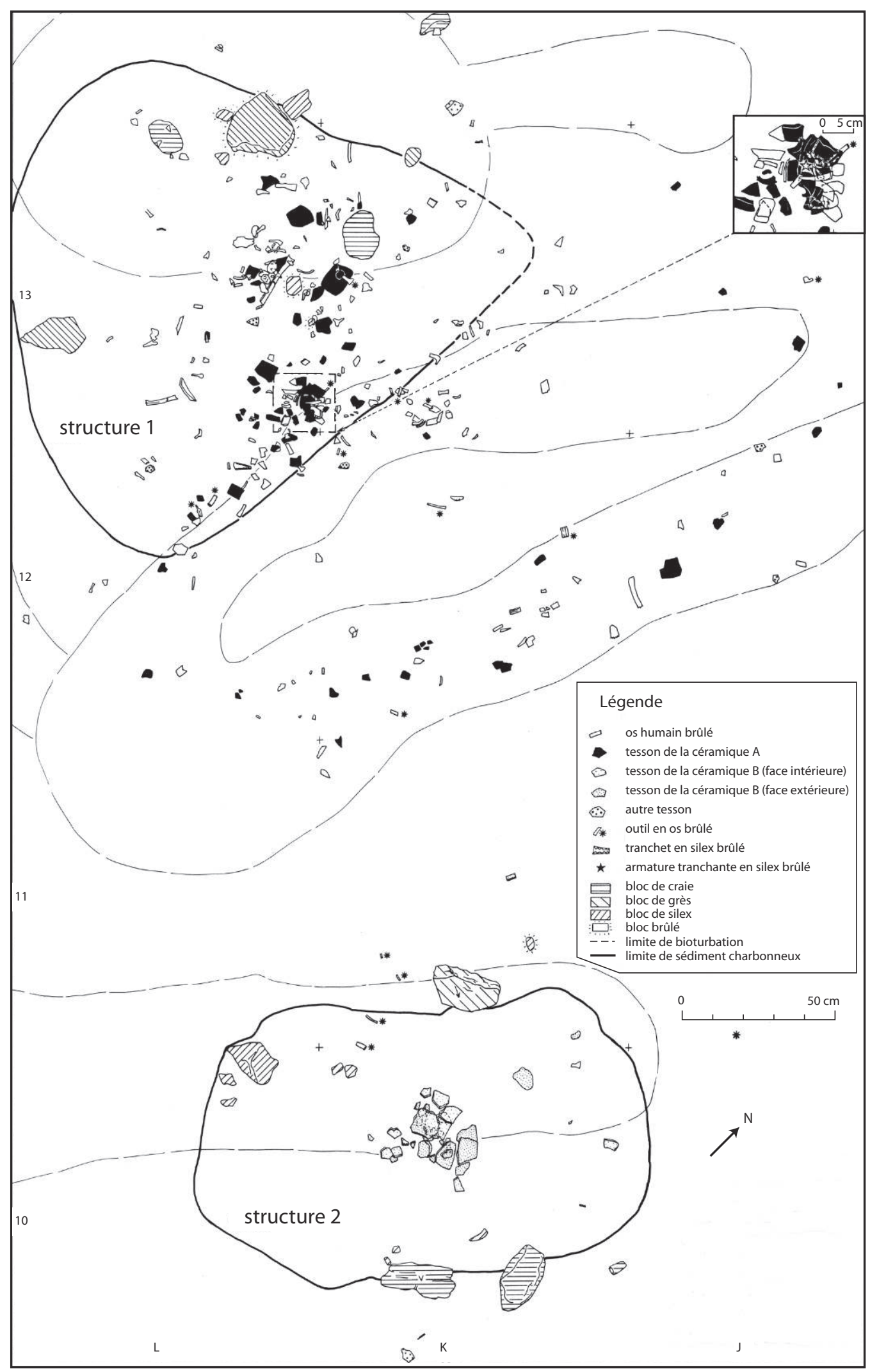

Figure 8 : Plan détaillé des vestiges des structures 1 et 2 .

Figure 8: Detailed plan of the remains of features 1 and 2 . 
L'industrie osseuse

L'industrie osseuse est à maints égards remarquable $\left(n^{\circ} 1\right.$ à 11, fig. 11 ; fig. 12). Elle comporte 16 fragments d'instruments apparentés à des poinçons ou à des pointes de sagaie. À l'instar du reste du mobilier, l'ensemble de ces fragments porte des traces de combustion (microfissures, esquillements, couleur blanc laiteux) et présente des courbures plus ou moins prononcées ainsi parfois que des torsions longitudinales. Les remontages réalisés entre fragments sont au nombre de cinq. Quatre d'entre eux sont situés en bordure de la structure 2 .

Les numéros 1 à 4 et 7 , fig. 11, peuvent appartenir à un même objet : pointe à soie de section aplatie et à feuille de section losangique; 5 et 9 peuvent appartenir à un second outil : poinçon de section carrée avec extrémité en biseau; 6 et 8 peuvent appartenir à un troisième objet proche du deuxième : poinçon à section subquadrangulaire et à extrémité en biseau. Les numéros 10 et 11 peuvent constituer un quatrième instrument: le premier fragment, ayant conservé l'articulation du métapode sur lequel l'instrument est façonné, est la base d'une grande lame présentant une nervure centrale le long de laquelle sont visibles des traces de façonnage (largeur de la lame : 1,7 cm); y sont également perceptibles deux fines entailles latérales pouvant être liées à un système d'emmanchement. Le deuxième fragment peut correspondre à la partie médiane du même objet, même si sa largeur est ici plus importante $(2,3 \mathrm{~cm})$ : la section est de forme voisine avec des traces de façonnage identiques le long de la nervure; la face opposée à la nervure est polie avec le même soin. L'objet aurait probablement eu une grande dimension, au minimum $25 \mathrm{~cm}$.

\section{La couche cendreuse de la structure 1}

Emprise et composition

Les ossements humains reconnus dans cette formation ne représentent que $544 \mathrm{~g}$ de fragments brûlés et se concentrent dans un angle de la partie excavée centrale, sur $2 \mathrm{~m}^{2}$. Aucun témoin d'une plus grande extension de cette couche à ossements n’a été découvert. En s'appuyant sur le plan de répartition général des vestiges de la structure 1 (fig. 8) ainsi que sur celui des remontages (fig. 9), plusieurs observations sont possibles.

- La très grande partie des éléments contenus dans la structure 1 est regroupée à la limite entre les carrés L13 et K13, sur une surface d'un demi-mètre carré. À cet emplacement, les vestiges sont associés par petits tas composés d'os incinérés (humains et animaux) et de tous les types de mobiliers, brûlés sans exclusion (tessons du récipient $\mathrm{A}$, fragments de poinçons, outils en silex, petits galets).

- On retrouve de manière dispersée ces mêmes éléments dans un rayon d'environ $2 \mathrm{~m}$ autour de la zone de concentration principale. Leur aire de répartition se superpose à l'emplacement de terriers, probables causes de la dispersion de vestiges initialement plus concentrés. Ils occupent généralement le fond des perturbations de sorte qu'ils se situent à un niveau nettement inférieur aux mobiliers concentrés en L13 et K13. Seul un grand creusement (probablement un terrier; fig. 5, st. 1 inf), situé immédiatement au nord-ouest de la structure 1, ne contient aucun de ces vestiges.

- La zone de dispersion des vestiges de la structure 1 s'inscrit entièrement dans les limites de la zone centrale quadrangulaire excavée.

- Quelques blocs de craie, de grès et de silex sont disposés autour des vestiges et peuvent représenter des témoins d'un aménagement de cette structure. Mais dans la mesure où ces blocs se situent dans l'angle occidental de la zone centrale, ils peuvent être également des vestiges de l'empierrement, basculés tardivement dans la partie excavée.

Cette nappe de vestiges osseux représente-t-elle l'intégralité du dépôt? Rien n'est moins sûr. Néanmoins, ces restes sont localisés sous le niveau d'empierrement et, de ce fait, ont pu être préservés des labours. Précisons également que presque aucune esquille appartenant à la couche osseuse n'affleurait au sommet du remplissage de la partie centrale du monument. Les effets des bioturbations sont par contre manifestes au sud-est de la structure avec la dispersion d'esquilles dans la galerie d'un terrier coudé.

\section{Identification des défunts}

Les éléments osseux de la structure 1 se composent exclusivement de restes incinérés, soit $544 \mathrm{~g}$ d'os humains (environ 283 pièces), auxquels s'ajoutent $74 \mathrm{~g}$ de faune (non inclus le mobilier osseux), brûlés avec la même intensité que les restes humains. Les éléments osseux sont dispersés dans l'ensemble de la fosse par petits paquets amalgamant os et mobiliers brûlés.

Trois individus ont été reconnus (cf. Duday, 1987). Le premier est représenté par au moins $19 \mathrm{~g}$ d'ossements; il s'agit de 13 portions de calotte crânienne dont la texture et l'épaisseur évoquent un sujet immature. Les deux autres, bien que de morphologie gracile, sont adultes (épiphysation de l'extrémité proximale de l'ulna; présence de ponts osseux, voire synostose de la table endocrânienne observée sur de petites portions de suture peut-être sagittale). Le nombre minimal de sujets adultes est apprécié grâce à deux extrémités proximales droites d'ulna. L'ensemble des restes donne une impression de gracilité : finesse des fragments de phalanges et $\mathrm{du} 5^{\mathrm{e}}$ métacarpien gauche, insertions musculaires peu marquées comme celle du deltoïde ou de la tubérosité conoïde, faible périmètre du radius (au milieu).

Les quelques caractères morphologiques observables sur le squelette céphalique correspondent à des critères féminins : arcades sourcilières peu dessinées, rebord sus-orbitaire de forme aiguë, petite mastoïde (Ubelaker, 1978), mais rappe- 


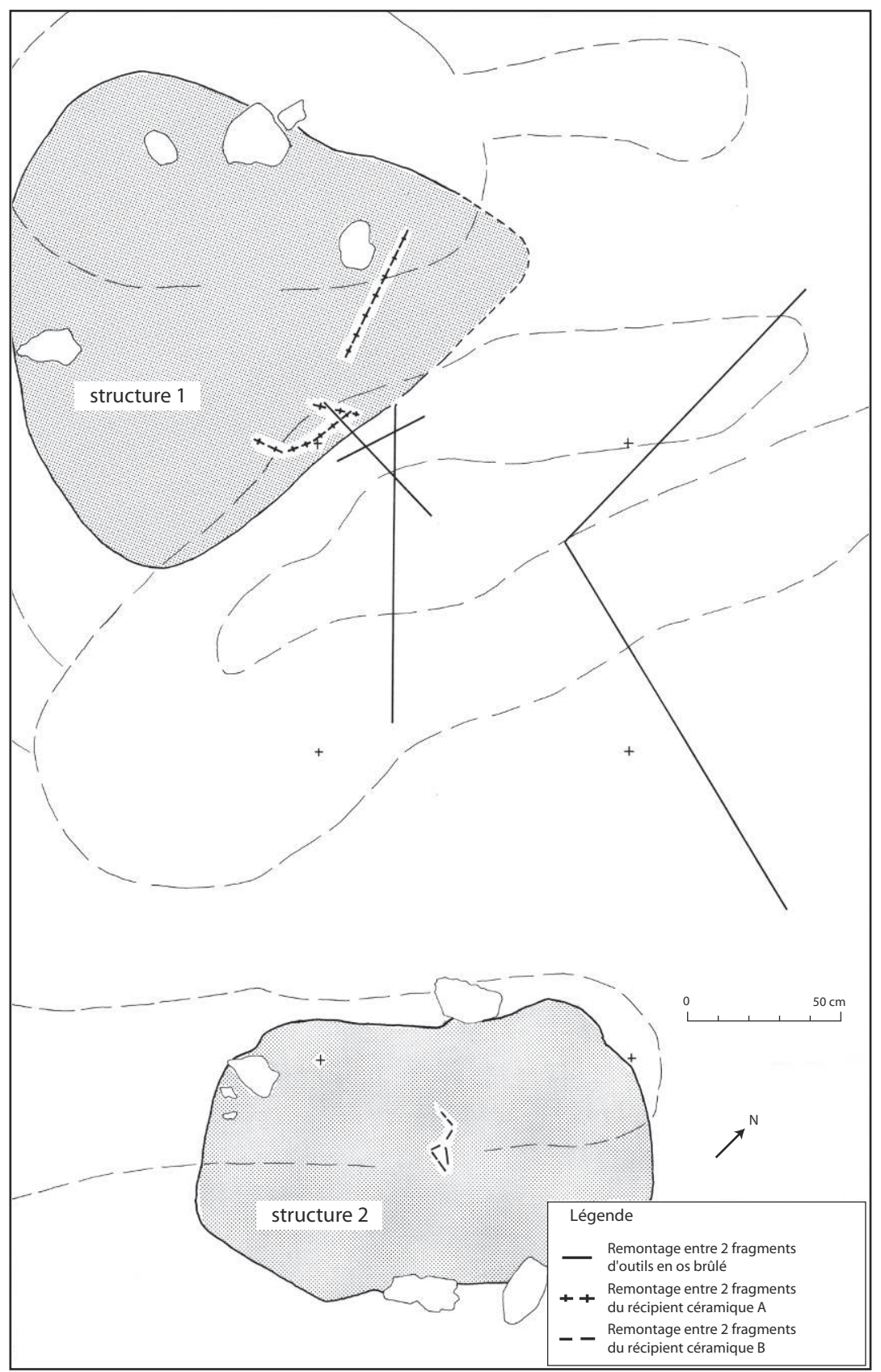

Figure 9 : Plan des liaisons spatiales par remontage du mobilier. Figure 9: Spatial links obtained by refitting artefacts.

lons qu'en l'absence des os coxaux il est délicat de déterminer le sexe du squelette.

\section{Répartition des individus}

Les fragments de calotte crânienne du sujet immature se répartissent sur plusieurs décimètres carrés, y compris dans le secteur non perturbé de la structure. Leur aire de répartition évoque un éparpillement et non pas un crâne écrasé ou brûlé sur place, ni un dépôt spécifique à ce sujet immature; en effet, les restes des sujets adultes lui sont étroitement associés (fig. 14). Il est en revanche impossible de discuter des aires de répartition de ces derniers. Comparables par leur morphologie gracile et brûlés avec la même intensité, nous ne disposons d'aucun indice ostéologique pour attribuer les pièces osseuses à l'un ou l'autre de ces sujets adultes. De même, l'analyse de la répartition des différents types d'os nous conduit à constater le mélange et la déstructuration de l'ordonnance anatomique des squelettes. 

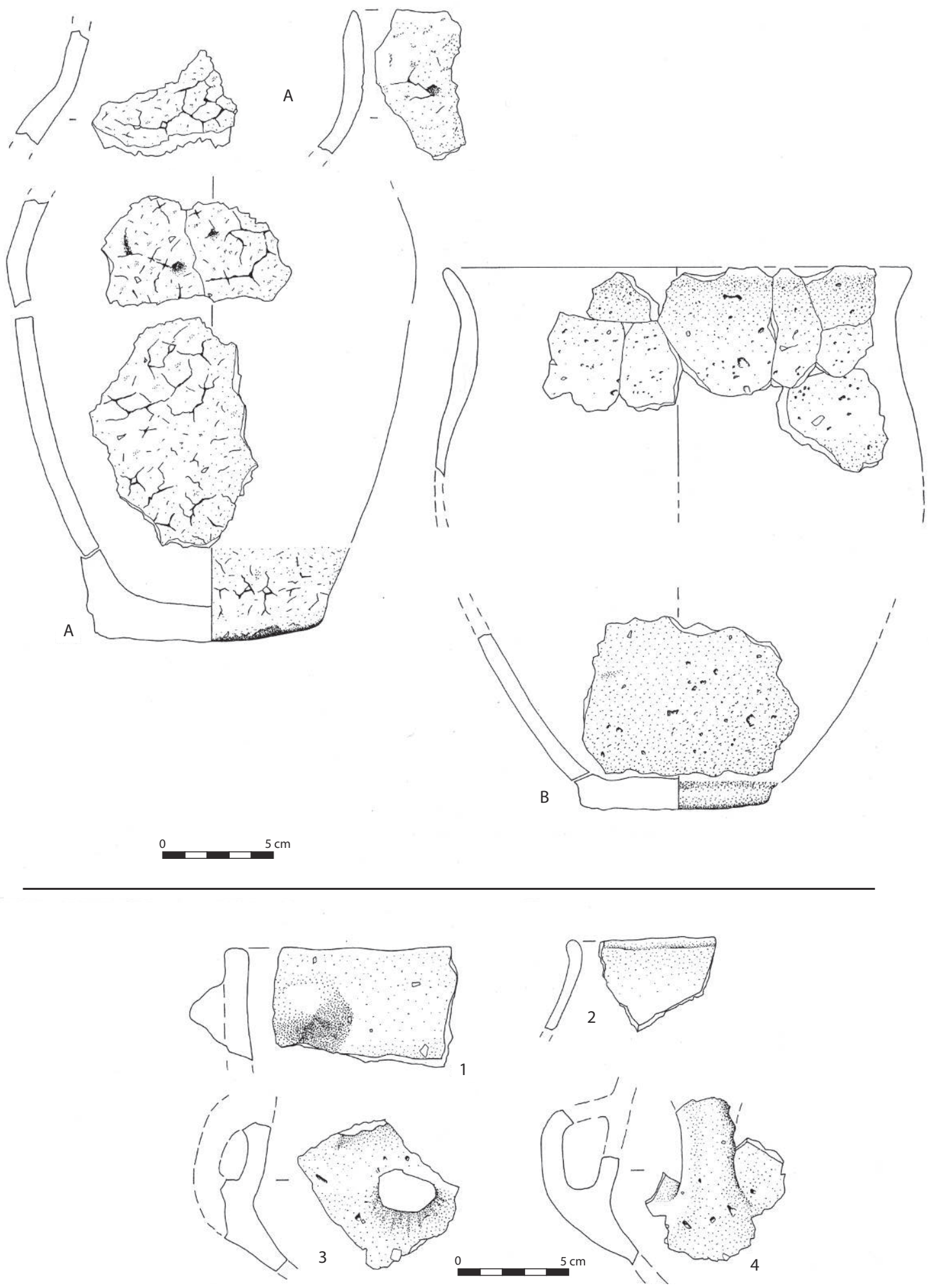

Figure 10 : Mobilier céramique; en haut à gauche, récipient $\mathrm{A}$ de la structure 1 (tessons très déformés par recuisson); en haut à droite, récipient $\mathrm{B}$ de la structure 2; en bas, éléments de formes céramiques provenant de la dépression périphérique (1à 3, concentration 2; 4 , concentration 1).

Figure 10: The ceramic assemblage; top left, vessel A from feature 1 (shards distorded by refiring); top right, vessel B from feature 2; below, caracteristic shards from the hollowed area surrounding feature 1 (1-3 from concentration 2; 4 from concentration 1). 

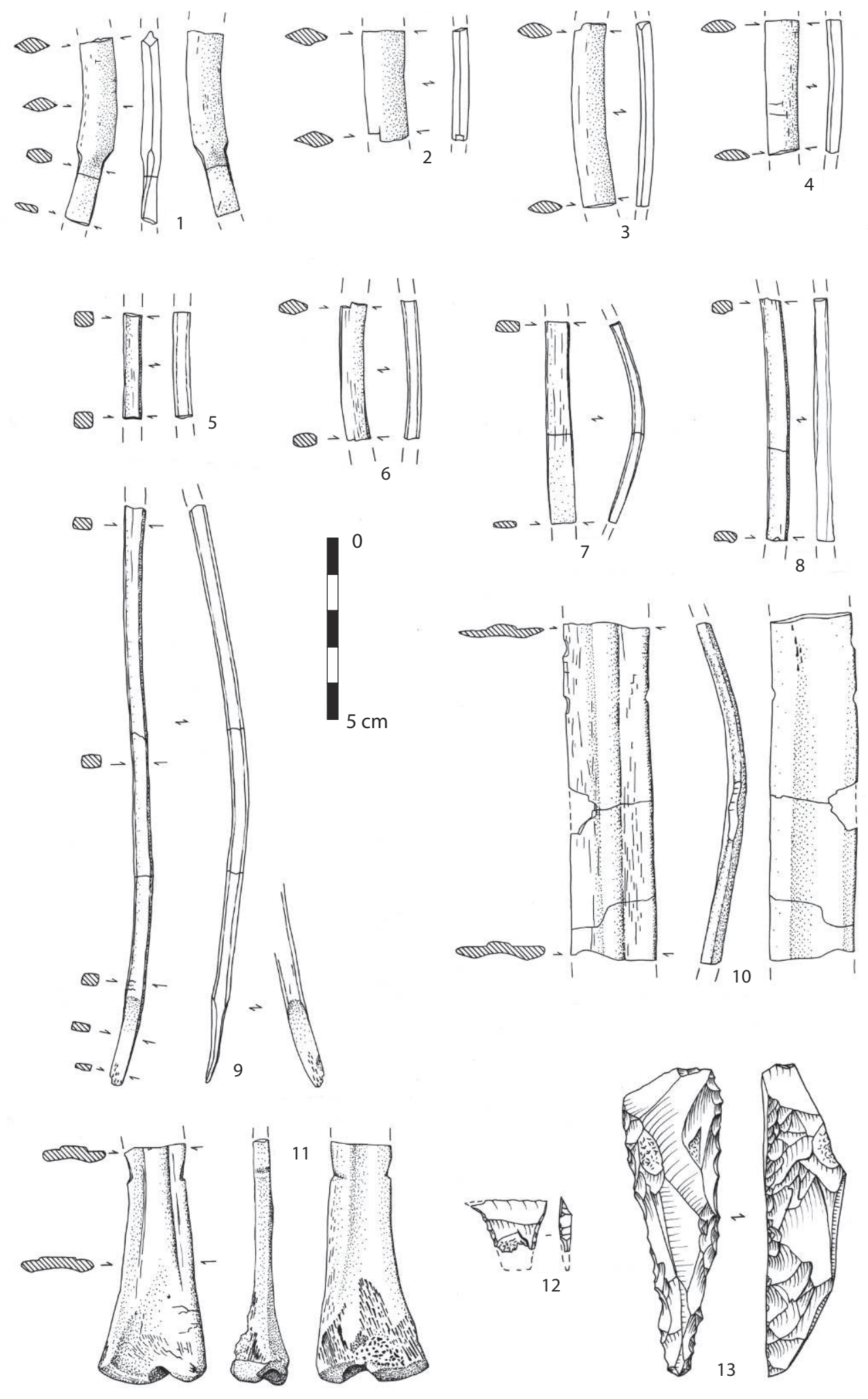

Figure 11 : Mobilier non céramique de la structure $1 ; 1$ à 11, industrie osseuse (fragments brûlés); 12, armature de flèche tranchante brûlée; 13 , tranchet brûlé.

Figure 11: Non-ceramic assemblage from feature 1; 1-11, bone industry (burnt fragments); 12, burnt transverse arrowhead; 13, burnt tranchet. 

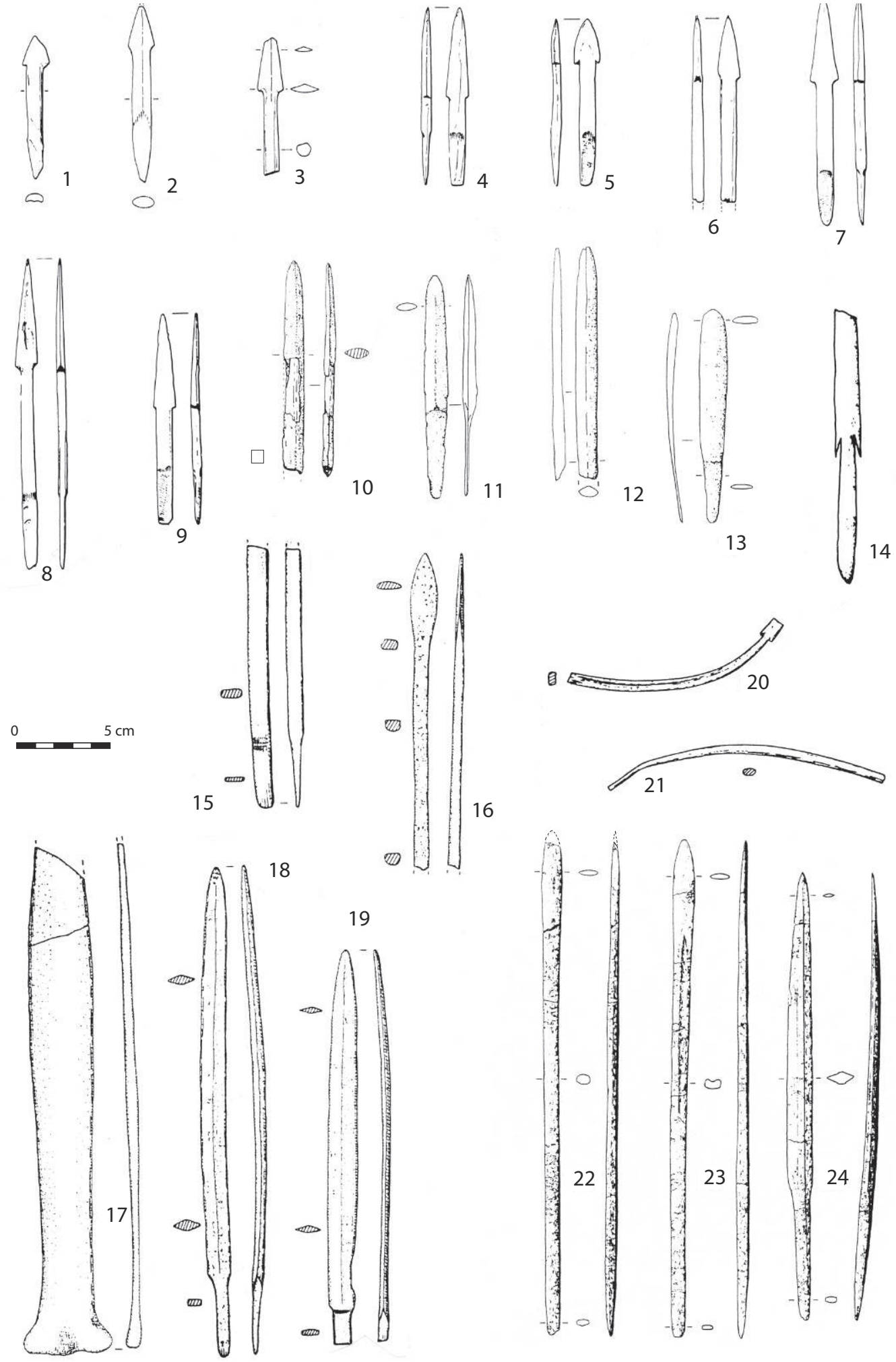

Figure 12 : Éléments de comparaison pour l'industrie osseuse; 1, Lohne (Allemagne) ; 2, Rimbeck (Allemagne); 3, Sorsum (Allemagne); 4 à 9, Schönstedt (Allemagne) ; 10, Coizard « Champ Machault ", grotte XVIII (Marne), coll. J. de Baye; 11, Oyes « La Butte du Moulin " (Marne, coll. de Baye; 12 et 13, hypogées de la vallée du Petit-Morin (Marne), coll. de Baye; 14, Crécy-en-Brie; 15 à 19, Isle-les-Meldeuse (Seine-et-Marne) ; 20 et 21, Stein (Pays-Bas); 22 à 24, Vignely "La Porte des Bergers » (Seine-et-Marne). 1 à 21 d'après Pape, $1982 ; 22$ à 24 d'après Allard et al., 1998.

Figure 12: Comparisons for the bone assemblage. 


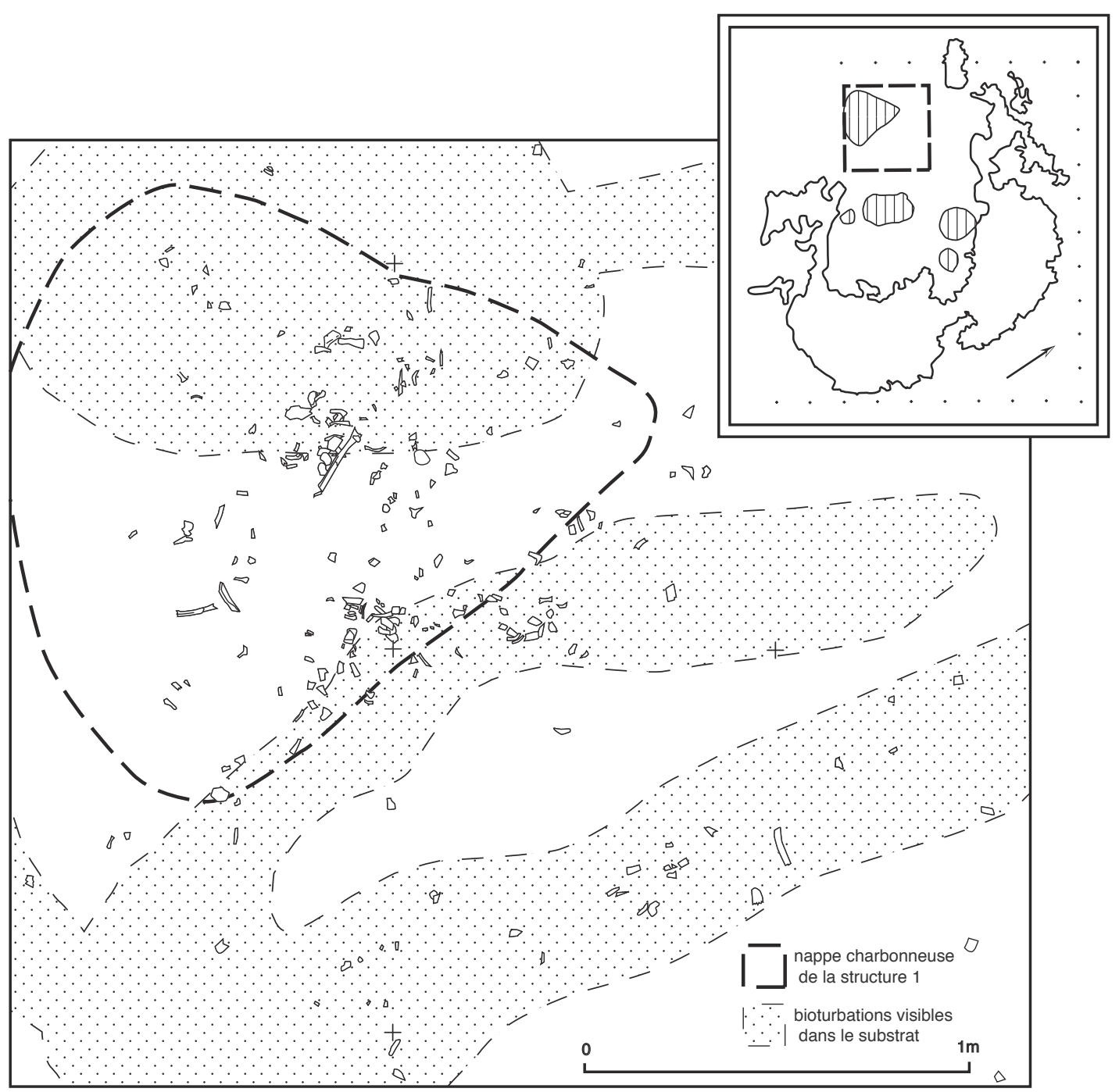

Figure 13 : Répartition des restes humains (brûlés) dans le monument.

Figure 13: Distribution of the human cremated remains in the monument.

Le squelette céphalique, le rachis ou les côtes sont en effet éparpillés sur l'ensemble de la fosse. Des fragments du maxillaire et de la mandibule sont distants de plus de $50 \mathrm{~cm}$. Les rares portions de diaphyses tibiales et de fibula sont également éloignées de plus de $60 \mathrm{~cm}$. Les pièces d'un même type d'os ou d'un même segment s'avèrent donc éparpillées. Il subsiste toutefois quelques pièces osseuses dont la proximité présente encore une cohérence anatomique : dans l'angle sud du carré K13, on notera la proximité de l'atlas, de portions d'occipital et d'un fragment de scapula gauche; on signalera encore, dans la moitié sud du carré L13, l'alignement selon un axe nord-sud, d'une longue portion de la diaphyse humérale droite, prolongée par un morceau de diaphyse radiale, qui jouxte plusieurs fragments d'ulna (os droit et gauche appartenant au même sujet) (fig. 14).
Représentation des différents secteurs du squelette

Les 554 grammes d'ossements recueillis dans la structure ne présentent assurément qu'une partie des vestiges de la combustion des trois défunts. En effet, le produit attendu de la crémation d'un corps de taille adulte est estimé, pour le moins, à $1000 \mathrm{~g}$ (Le Goff, 1998). Bien que l'on constate, au travers de la littérature spécialisée, l'importance des variations du poids d'un squelette non brûlé - par comparaison avec le poids moyen des séries de squelettes asiatiques (Lowrance et Latimer in Krogman et Iscan, 1986), il est de $2882 \mathrm{~g}$ - on mesure quelque peu l'importance de la part manquante.

En outre, les membres inférieurs ainsi que les pieds, avec $46 \mathrm{~g}$ d'os seulement, s'avèrent comparativement sous-représentés. Le poids moyen de ce secteur du squelette, d'après les données sur squelettes secs et complets publiées par 


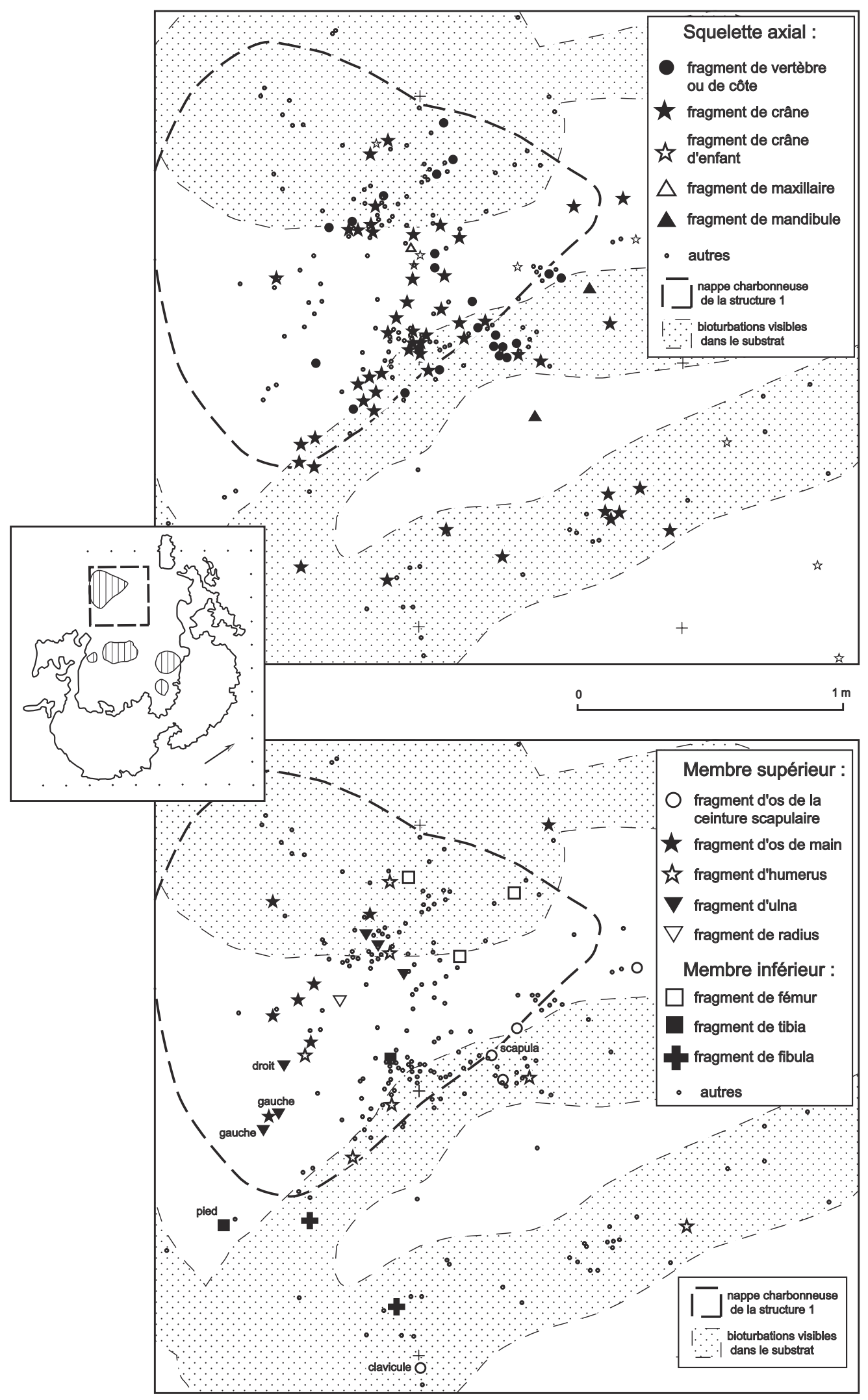

Figure 14 : Répartition des ossements par régions anatomiques.

Figure 14: Distribution of the bones, by anatomy. 
Krogman (in Krogman et Iscan, 1986) est estimé à $905 \mathrm{~g}$ (fig. 15 A et B). En revanche, on note une forte sur-représentation du squelette céphalique : avec $237 \mathrm{~g}$, il représente presque $50 \%$ du dépôt de la structure 1 alors que cette partie ne constitue que $20 \%$ du poids d'un squelette complet. L'ensemble des vestiges osseux des défunts ne figure donc plus dans la structure 1 et les proportions anatomiques ne sont plus cohérentes.

Le stade de crémation

La couleur des esquilles atteste que l'incinération s'est poursuivie jusqu'à l'obtention d'ossements blancs. Il s'agit d'une opération menée jusqu'à complète disparition des chairs et carbonisation des ossements impliquant la mise en œuvre d'un feu intense ou suivi et entretenu. Tous les secteurs du corps ont atteint le même stade de combustion sauf quelques rares pièces plus épaisses comme la mastoïde, de couleur gris foncé ou quelques portions gris clair de la diaphyse fémorale.

L'intensité de la combustion de la matière osseuse est également perceptible par la texture cristalline des os. On notera la déformation des portions de diaphyses humérales (courbure ou enroulement) et celle de la fibula, sans toutefois produire de fissures transversales. Les fissures longitudinales s'avèrent également rares. Quelques fissures en forme de lunule parcourent deux ou trois portions de calotte crânienne. Du point de vue du mode de fissuration, les pièces osseuses présentent peu des indices interprétés habituellement comme des indicateurs de crémation d'os frais (Buiskra et Swegle, 1989). Du point de vue des déformations, certaines portions de diaphyses notablement courbées évoquent en revanche des critères de l'incinération d'os frais. a "structure 2 "

Description générale

La « structure 2 » a été identifiée par l'apparition à la fouille d'une tache de sédiment brun noir légèrement charbonneux, de forme grossièrement ovale et délimitée par des blocs de pierre. Au centre de ce qui semble être une fosse est apparue une concentration de tessons appartenant à un unique vase. Même si l'ensemble des tessons est loin d'avoir été conservé, leur dispersion est beaucoup moins grande que celle des vestiges de la structure 1 puisque la quasi totalité des fragments se trouve dans les limites de la structure et plus précisément dans son centre. Quelques petits galets brûlés, pour un poids total de $900 \mathrm{~g}$, sont présents dans la fosse et tout particulièrement à l'emplacement de la concentration de tessons.

Le remplissage de la fosse n'est pas homogène puisque, à certains endroits et en particulier dans les parties sud et nord, sont présentes des poches de sédiment très charbonneux aux contours assez flous (fig. 3). Leur présence doit être mise en relation avec l'aménagement de blocs disposés de chant autour de la fosse. Deux autres blocs de forme plus globuleuse sont également présents en limite de la fosse à l'est et à l'ouest. L'ensemble de ces éléments peut laisser présager l'existence d'une structure en bois de type coffre. Il faut néanmoins rester prudent à cause d'importantes perturbations : la structure 2 a très probablement été affectée par les deux terriers (structures 7 et 8 , fig. 5) dont on peut observer la base à un niveau inférieur. La présence de sédiment charbonneux dans la fosse peut résulter du colmatage différé d'une structure en bois et ce sédiment proviendrait alors d'une structure de combustion postérieure ou tout du moins recouvrant la structure 2 . La présence de galets brûlés à l'emplacement du récipient tend néanmoins à montrer une relation entre le dépôt mobilier et une activité de combustion.

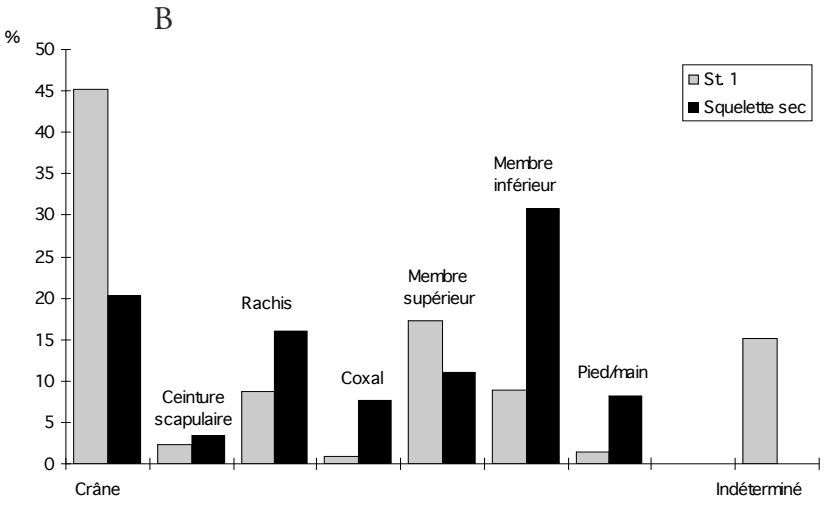

Figure 15 : A - Structure 1, poids des os des sujets adultes comparé au poids moyen d'un squelette sec (d'après Krogman et Iscan, 1986); B - Structure 1, proportion des différentes parties du squelette recueillies par comparaison avec celles d'un squelette sec complet. Figure 15: A - Feature 1, weight of the adult bones collected, compared to the average weight of dry bone; $B$ - Feature 1, proportion of the different skeleton parts collected, compared to a complete skeleton. 
La céramique

Les 48 tessons recueillis dans la structure 2 appartiennent à un unique vase (récipient $\mathrm{B}$, fig. 10) et sont de taille moyenne, les plus gros atteignant $10 \mathrm{~cm}$ de module. La pâte a une couleur homogène gris vert, à la fois à l'intérieur, à l'extérieur et en section. Le dégraissant est grossier mais peu abondant (os, chamotte). De nombreuses alvéoles montrent qu'une grande partie du dégraissant a disparu. Les tessons sont recouverts d'une fine pellicule marron gris, incrustée dans la pâte et qui ne peut s'enlever sans altération de son état de surface.

Comme dans la structure 1 , le très faible taux de remontage (6 cas seulement) est dû à l'érosion des tranches. L'altération naturelle de la céramique dans le sédiment est importante et a surtout touché la face supérieure du tesson, exposée aux intempéries. Ce vase $B$ n'a pas subi les déformations observées sur le récipient $\mathrm{A}$, mais des indices de recuisson pourraient résider dans l'altération générale des tessons et surtout dans la disparition complète de toute matière organique à l'intérieur de la pâte, phénomène déjà observé sur le récipient $\mathrm{A}$.

Le manque de remontages empêche de restituer le détail de la forme de la panse, mais le profil général est mieux assuré que pour le récipient $\mathrm{A}$. Il s'agit également d'un vase simple à fond plat et probablement à profil en $S$, car aucun tesson ne suggère de segmentation du profil. Son ouverture mesure environ $22 \mathrm{~cm}$ pour une hauteur estimée à $25 \mathrm{~cm}$. L'absence d'élément de préhension et de décor est un point commun avec le récipient $\mathrm{A}$. Le bord est aminci et légèrement évasé. L'épaisseur du fond est de $13 \mathrm{~mm}$ et celle de la panse est de $8 \mathrm{~mm}$.

\section{Mode de dépôt}

Contrairement à la structure 1, la structure 2 n'a livré aucun reste osseux ni aucun autre mobilier, mis à part quatre petits fragments d'outils en os et deux esquilles d'os humain brûlé qui ont de très fortes chances de provenir de perturbations de la structure 1. Néanmoins, l'aspect de la céramique rejoint la présence de témoins de combustion (petits galets chauffés et sédiment charbonneux) dans l'hypothèse d'une association de la structure 2 à une pratique de combustion.

\section{Les autres structures}

Lentilles et fosses

D'autres structures à remplissage sombre ou charbonneux (st. 2 bis, 3 et 10) se répartissent dans les limites de l'empierrement (cf. fig. 3). Aucune d'entre elles n'a livré de mobilier.

- La structure 2bis est une petite lentille de sédiment sombre sans charbon, située dans la partie supérieure du colmatage de la dépression centrale.
- La structure 3 est également une fine lentille de sédiment brun noir, qui n'est apparue que dans le milieu du remplissage. Elle est délimitée dans sa partie sud par deux petits blocs calcaires.

- La structure 10 est une fosse de presque $1 \mathrm{~m}$ de diamètre et $50 \mathrm{~cm}$ de profondeur, atteignant le substrat de la partie excavée. Elle apparait dès le sommet du remplissage et se signale par son contenu très charbonneux, avec dans sa partie centrale une grosse concentration de charbons. Quelques blocs de craie figuraient également dans la fosse.

\section{Bioturbations et fréquentations tardives}

Le remplissage de ces différentes perturbations est souvent vide de vestiges, sauf aux alentours immédiats de la structure 1 où elles contiennent quelques ossements brûlés, fragments d'outils en os et tessons du récipient A. Le déplacement de ces vestiges en dehors de la structure 1 va dans le sens de l'hypothèse de leur remaniement par des fouisseurs.

Parmi ces perturbations, on peut distinguer des creusements linéaires, peu profonds et qui sont les plus nombreux. Certains (st. 4, 5 et 7) semblent passer sous l'empierrement sans vraiment le perturber (exceptée pour la st. 4). Cette donnée est particulièrement intéressante car elle laisse à penser que l'obstacle qu'a pu constituer le parement de craie a contraint les animaux fouisseurs à creuser sous le niveau de l'empierrement. L'espace de circulation des fouisseurs aurait donc été limité, en haut par la présence du parement et en bas par le substrat graveleux.

Deux grands creusements (st.1 inf. et 8) se distinguent du reste des perturbations. Il s'agit de fosses ovales, stériles, très profondes et jouxtant les structures 1 et 2 sans les remanier. Des couloirs de terriers menant à ces fosses, on peut penser que l'on a affaire à des gîtes d'animaux fouisseurs, mais l'hypothèse de structures anthropiques ne peut être exclue.

Par ailleurs, en périphérie de la zone empierrée, on a pu relever dix fosses (st. 11 à 20), localisées à la fois dans et à l'extérieur de la grande dépression (fig. 2). Pour l'essentiel, elles n'ont pas livré de mobilier à l'exception des structures 11 et 13 . Quatre éléments de formes céramiques permettent de les rattacher à une occupation du Bronze final, déjà attestée sur le reste du site par la présence de fosses éparses (Langlois et Celly, 1995).

\section{LeS ANALYSES PALYNOLOGIQUeS}

Deux prélèvements palynologiques ont été réalisés : le premier échantillon a été prélevé en J7, dans le niveau humifere immédiatement sous-jacent à l'empierrement de blocs de craie, c'est-à-dire dans le paléosol néolithique antérieur à l'édification de la structure funéraire, à un endroit où cet 
empierrement était particulièrement épais. Le deuxième a été pris dans le fond de la structure 2 (cf. fig. 4). Ils ont été analysés par F. Reckinger et A. V. Munaut (Université de Louvain). Les résultats de ces analyses peuvent être comparés à la fois à ceux portant sur les structures du VilleneuveSaint-Germain et à ceux se rapportant à La Tène ou au galloromain (tableau 1 ; Bostyn [dir.], 2003).

Le spectre pollinique du paléosol montre une prédominance très nette des composées de type Crepis; il est plus proche des spectres de La Tène et de la période gallo-romaine que de ceux des fosses du Néolithique ancien du même site. Ceci peut être expliqué par le caractère très perturbé (terriers, lombrics...) de ce niveau.

Léchantillon prélevé dans la structure 2 elle-même donne un spectre légèrement moins déboisé $(\mathrm{AP}=8,1 \%)$, mais avec une prédominance de composées de type Crepis moins forte que dans les autres fosses VSG. Les pollens céréaliens sont abondants $(10,7 \%)$ mais la particularité de ce spectre réside dans le taux assez élevé de brassicacées (22,2\%). Les pollens de cette famille se ressemblent tous et il est malheureusement impossible de distinguer les espèces auxquelles ils appartiennent. Les fleurs de cette famille sont souvent assez grandes et colorées; il n'est donc pas exclu que ces pollens proviennent de végétaux déposés en offrandes.

\begin{tabular}{|c|c|c|}
\hline & Paléosol & Structure 2 \\
\hline Alnus & 0,6 & 0,9 \\
\hline Betula & 0 & 1,3 \\
\hline Corylus & 1,2 & 2,1 \\
\hline Pinus & 0,2 & 3 \\
\hline Quercus & 0,6 & 0,9 \\
\hline Tilia & 0,3 & 0 \\
\hline \%AP & 3,7 & $\mathbf{8 , 1}$ \\
\hline Apiaceae & 0,3 & 0 \\
\hline Brassicaceae & 1,5 & 22,2 \\
\hline Centaurea scabiosa & 0 & 0,4 \\
\hline Cerealia & 4,3 & 10,7 \\
\hline Secale cereale & 0 & 0,4 \\
\hline Chenopodiaceae & 0,6 & 0 \\
\hline Cirsium & 0 & 0,4 \\
\hline Crepis & 79,9 & 42,3 \\
\hline Cyperaceae & 0,3 & 0 \\
\hline Plantago lanceolata & 1,2 & 0,9 \\
\hline Plantago major & 0 & 0,4 \\
\hline Poaceae & 7,6 & 13,2 \\
\hline Ranunculaceae & 0 & 0,4 \\
\hline Urtica & 0,3 & 0 \\
\hline Dryopteris & 0 & 0,4 \\
\hline Pteridium aquilinum & 0,3 & 0 \\
\hline \%NAP & $\mathbf{9 6 , 3}$ & $\mathbf{9 1 , 9}$ \\
\hline Total Pollens & 328 & 234 \\
\hline
\end{tabular}

Tableau 1 : Étude palynologique des deux échantillons du monument de Poses «Sur la Mare ».

Table 1: Pollen results from the two studied samples.
Rappelons que dans le cairn du Néolithique moyen de Vierville (Manche), une concentration anormale de pollens de tilleul (27\%) et de Reine des Prés (Filipendula) (49\%) a été trouvée sous un crâne, alors que ces espèces n'ont pas été retrouvées dans le paléosol, ni dans un environnement plus éloigné (Clet-Pellerin, 1986). Dans ce dernier cas, l'hypothèse d'une pratique funéraire utilisant une litière et/ou une parure végétale a été avancée.

\section{SYNTHÈSE}

\section{Architecture et fonctionnement}

L'édifice de Poses présentait probablement un tumulus de plan ovalaire, dont au moins la base était empierrée. La partie centrale, que nous avons appelée « la chambre " par commodité, était légèrement surcreusée d'environ 20 à $30 \mathrm{~cm}$ et on peut supposer qu'elle était occupée par une structure bâtie en bois. Aucun trou de poteau ne vient cependant à l'appui de cette hypothèse mais la présence de deux " antennes » à la limite sud-est de la partie centrale pourrait évoquer l'utilisation de deux sablières basses en bois, situées le long des deux grands côtés. La forme quadrangulaire de la partie centrale et l'absence de fosses de calage d'orthostates permettent en tout cas d'exclure une construction mégalithique ou en encorbellement. L'état de conservation du site lors de la fouille n'a pas permis d'observer de système d'accès à cette " chambre ". La partie occidentale de l'empierrement semble nettement plus arasée et, de ce fait, toute observation à ce propos y est exclue.

L'existence de la grande dépression périphérique à l'empierrement est une donnée exceptionnelle, qui s'explique très probablement par l'aménagement d'une masse tumulaire. Les données de la fouille ne peuvent contribuer à restituer le volume d'un tel tumulus, mais rendent néanmoins crédible cette hypothèse. En effet, la dépression périphérique mesure près de $414 \mathrm{~m}^{2}$ (fig. 2). L'estimation de la surface au sol de l'édifice est d'environ $70 \mathrm{~m}^{2}$ et celle de la zone centrale d'environ $24 \mathrm{~m}^{2}$. Si l'on suppose que les sédiments prélevés dans cette dépression correspondent à une épaisseur voisine de 20 à $40 \mathrm{~cm}$, leur volume pourrait atteindre entre 83 et $165 \mathrm{~m}^{3}$, ce qui semble largement suffire à couvrir la totalité de cette structure funéraire. Ce calcul ne repose toutefois que sur des approximations et ne peut aboutir à des données concrètes, d'autant que l'association stricte entre l'édifice et la cuvette périphérique est probable mais non démontrée. De plus, ni la part exacte des blocs de craie utilisés dans son édification ni son élévation ne sont connues. L'épaisseur de sédiment raclé autour de l'empierrement est également difficile à restituer précisément.

La nature exacte du fonctionnement d'un tel monument est également délicate à évoquer. Deux questions méritent 
d'être distinguées : celle de la nature des dépôts sépulcraux et celle du mode de dépôt funéraire.

\section{La nature des dépôts sépulcraux}

La première question consiste à savoir si le monument a constitué un lieu de dépôt primaire (un caveau) ou bien le réceptacle d'ossements décharnés dans un autre lieu (un ossuaire). Malgré un surcreusement important de la " chambre ", aucune trace de dépôts primaires n'a pu y être identifiée. Si l'édifice avait accueilli une couche sépulcrale comportant des corps entiers, il est fort probable que, même dans l'hypothèse d'un enlèvement des ossements, certains des plus petits d'entre eux auraient été retrouvés sur place, ce qui n'est pas le cas. On ne peut toutefois exclure un nettoyage minutieux des ossements ou bien l'utilisation de contenants organiques pour le décharnement sur place puis le transport d'un faible nombre d'individus vers le lieu de la crémation.

Une autre hypothèse pouvant expliquer l'absence d'ossements non brûlés est l'arasement par les labours d'une couche sépulcrale située à l'origine à une altitude supérieure. Cette hypothèse est peu plausible dans la mesure où elle suppose un colmatage rapide de la zone centrale excavée et un niveau de dépôt nettement plus élevé que le sol néolithique. De plus, l'organisation de la structure 1 en nappe de vestiges évoque davantage un épandage sur un sol qu'une structure en creux.

Autant dire que les arguments favorables à l'hypothèse d'un lieu de dépôt secondaire sont plus convaincants, mais loin de permettre un diagnostic catégorique et définitif. Aussi avons-nous développé ici trois hypothèses.

\section{Incinération sur place?}

En premier lieu, il est difficile de trouver les indices d'un feu allumé sur place dans la partie centrale du monument. Les témoins de combustion restent limités aux vestiges mobiliers de la structure 1 et à la composition charbonneuse du contenu de la plupart des autres fosses. Une incinération dans le monument aurait marqué l'architecture, d'autant plus que l'exposition des corps au feu a été manifestement très poussée. Si l'on en juge par les descriptions de monuments qui contiennent des corps brûlés sur place (Lacroix-SaintOuen, Barbonne-Fayel, Lignon, Ribemont...), l'altération des parois de pierres du pourtour interne des caveaux et du sédiment du sol s'y avère remarquable. Or, ici, le contraste entre des témoignages d'une forte altération thermique des os et l'absence de traces de feu dans la " chambre " est saisissant. C'est là le principal contre-argument à l'hypothèse d'une combustion des corps sur place.

Quant à la documentation ostéologique, elle illustre le brassage des os et la perte de l'individualité des sujets. La dis- position de quelques pièces osseuses évoque simplement un membre supérieur en extension, cohérence anatomique bien modeste au regard des exemples de crémation de Stein ou de Lacroix-Saint-Ouen, où le corps des défunts est retrouvé dans une posture identifiable à l'issue de la combustion.

Lincinération d'un corps sans intervention des opérateurs n'induit pas à elle seule la déstructuration des squelettes. En effet, la fouille de la sépulture collective SOM de Lacroix-Saint-Ouen (Oise) a montré que la position initiale des corps, même après crémation des os et incendie de la sépulture, demeure tout à fait lisible. Il subsiste encore de nombreuses connexions (Guillot et Le Goff, 1995; Le Goff et al., 2002). Le squelette de Saint-Antoine, brûlé lors de l'incendie d'une maison vers 180 de notre ère, témoigne également de la persistance des connexions et de la position du corps (Haldimann et al., 1991). On citera encore ici l'exemple de la fosse du Néolithique final de Reichstett (BasRhin), qui a livré les vestiges d'une crémation primaire d'au moins 11 individus (Blaizot et Boës, 2003).

Dans la structure 1 de Poses, l'agencement des os implique un mélange des restes des trois corps de sorte que, dans l'hypothèse d'une crémation in situ, les corps ne se trouvent plus dans la posture donnée lors de l'allumage du feu. Il est probable, en raison du faible poids des restes augurant de prélèvements, qu'il ne s'agisse plus de la position des vestiges à l'issue de la combustion.

Nous n'avons guère plus d'arguments en faveur d'une combustion des corps survenue à l'extérieur du monument, de sorte que celui-ci ferait partie des quelques sépultures collectives qui abritent des incinérations. Cette hypothèse soulève la question du vocabulaire employé.

Pour des os brûlés issus de l'incendie d'un monument peut-on utiliser le terme "crémation "? Issu du mot latin cremare, son emploi dans des situations particulières (brûler une ville, brûler une victime offerte en sacrifice ou un supplicié...) confère à l'action de brûler des cadavres une vocation rituelle. Nous avons fait le choix d'utiliser plutôt ici le terme « incinération " car il n'est pas réservé à la combustion des corps en tant que pratique funéraire. Il est également employé en médecine et son usage est élargi par ailleurs à d'autres domaines comme celui du traitement des ordures ménagères. Dans l'hypothèse d'une combustion en dehors du monument funéraire, peut-on utiliser le terme " crémation "? Il supposerait l'existence d'un bûcher funéraire destiné à la combustion du corps ce dont nous n'avons aucune certitude. En tous les cas, il est délicat de montrer que le feu a été utilisé en vue de modifier l'architecture du caveau comme cela est le cas par exemple pour l'allée sépulcrale mégalithique de La Chaussée-Tirancourt (Oise) où la réfection du lieu a engendré une légère altération thermique de quelques os (Masset, 2002). Ici, le feu n'a pas non plus 
été employé à des fins de destruction comme l'illustrent les sépultures de Lacroix-Saint-Ouen (Oise) ou de La Hoguette à Fontenay-le-Marmion (Calvados).

\section{Dépôt secondaire (en urne ou déversé)?}

Dans le cas de la structure 2 , le dépôt du récipient $B$ à l'intérieur d'un aménagement en coffre est probable. Mais l'absence d'ossements humains brûlés associés pose problème pour interpréter ce vase en tant qu'urne cinéraire. Toutefois la présence de petits galets brûlés lie sa mise en place à l'usage du feu.

Dans le cas de la structure 1, l'ensemble des vestiges mobiliers a apparemment fait l'objet d'un dépôt simultané au même endroit. Se pose alors la question de leur mode de dépôt. Un récipient a pu servir de réceptacle. $\mathrm{La}$ "recuisson » et la forte fragmentation du récipient $\mathrm{A}$ laissent à penser que l'objet a participé à la crémation des corps mais que son état à l'issue de l'opération n'était plus compatible avec la fonction de contenant. Il n'est d'ailleurs pas totalement établi que ces tessons n'appartiennent qu'à un seul récipient. Celui-ci, trop déformé et résiduel, n'a pu servir de réceptacle aux quelque $600 \mathrm{~g}$ d'os brûlés et aux reliquats d'objets retrouvés.

Aussi est-il envisageable que le mode de dépôt puisse résulter du transfert des vestiges mélangés de la combustion d'un bûcher vers la « chambre ". L'opération aurait concerné os, mobilier et résidus de combustion sans qu'aucun tri ni aucune action ciblée sur les vestiges humains ne soit décelable. On constate alors que le monument reçoit un dépôt osseux peu centré sur les morts et que les restes ne sont pas individualisés, de sorte qu'il a pu fonctionner comme un ossuaire. Il n'est pas le lieu de traitement des corps mais un espace de dépôt des restes physiques des défunts, contrairement au modèle actuellement proposé pour les tombes collectives du Néolithique final (Chambon, 2003). De plus, les os des trois défunts ont pu être transférés dans le sépulcre en un même geste et non successivement, ce qui n'évoque plus le mode de fonctionnement d'une sépulture collective.

\section{Nettoyage de la couche osseuse par le feu?}

En dernière hypothèse, on peut avancer un fonctionnement de type vidange de la couche osseuse avec réduction des restes par le feu. Sur les autres sites attestant d'une vidange, les ossements sont très mal brûlés (argument principal) et associés à d'autres ossements non brûlés. Ils sont fréquemment relégués dans un coin, contre la paroi ou au contraire regroupés en tas. Dans le cas de "l'ossuaire " de Berry-au-Bac par exemple (Chambon, 1995), les ossements brûlés représentent seulement $7 \%$ du total. Cette hypothèse se heurte à nouveau au contraste entre le stade de créma- tion des os et l'absence de traces de feu dans le monument. Comment expliquer que l'énergie thermique déployée pour brûler les cadavres ait si peu marqué le sol ou le parement du cairn?

En revanche, la disposition des vestiges - dispersion des fragments d'un même os, absence des membres inférieurs, mélange dans une matrice sédimentaire d'une partie d'un produit de la crémation des trois individus et du mobilier - évoque des gestes déstructurants, comparables à ceux réalisés au cours d'une vidange. Un geste semblable est observé à Saint-Laurent-sur-Oust (Morbihan), dans la sépulture à entrée latérale de Beaumont (Tinevez, 1988; Tinevez et al., 1990), où des restes osseux brûlés sont associés à des objets brisés par le feu (lames et haches polies en silex). Dans les deux cas, on notera le faible nombre d'individus, trois à Saint-Laurent-sur-Oust également. Si, sur ce site, la nature géologique du substrat explique la disparition des os inhumés, il n'en va pas de même à Poses, où l'on observe l'absence de vestiges, même résiduels, des squelettes inhumés. Est-ce le résultat d'un curage soigné de la couche osseuse?

\section{Chronologie et comparaisons}

\section{Les datations}

Le monument a donné lieu à deux datations ${ }^{14} \mathrm{C}$. La première a été réalisée sur un lot de charbons provenant d'une poche délimitant la structure 2. Nous avons vu qu'elle pourrait correspondre à un aménagement de coffrage détruit par le feu. La datation obtenue est : Lyon-446 (OxA) : $4435 \pm 40$ BP, soit en datation calibrée [3301-2930] avant J.-C.

La seconde analyse a pu être réalisée dans le cadre d'un programme expérimental mis en place par Jan Lanting à l'université de Groningen. Il s'agit des premiers essais visant à élaborer une nouvelle méthode de datation du carbone minéral, lorsque la fraction organique de l'os (le collagène) a disparu soit lors d'une combustion de l'os, soit dans des conditions climatiques arides (sépultures de la zone sahélienne) (Saliège et al., 1998; Person et al., 1998; Lanting et Brindley, 2005). Le protocole mis en place a porté sur des sites pouvant livrer à la fois des charbons de bois et des restes osseux brûlés. Depuis ces premières expérimentations, la méthode a montré sa validité. L'analyse effectuée sur un os brûlé de la structure 1 de Poses a livré la date GrA-14811 : $4770 \pm 40 \mathrm{BP}$, soit [3644-3381] avant J.-C. en datation calibrée. Cette deuxième date est sensiblement plus ancienne que la première et un intervalle de 80 ans sépare les deux fourchettes. 


\section{Le mobilier}

Le mobilier archéologique est conforme à une datation ${ }^{14} \mathrm{C}$ calibrée vers la fin du quatrième millénaire. Les récipients A et $\mathrm{B}$ n'autorisent pas vraiment de comparaisons fines du fait de leurs reconstitutions partielles. On peut considérer qu'ils se placent dans un intervalle allant du Néolithique récent à l'âge du Bronze. Nous sommes néanmoins tenté de rapprocher le vase de la structure 2, caractérisé par un profil en $S$ et un épaulement relativement marqué, des offrandes céramiques du complexe funéraire Seine-OiseMarne (en tant que phase ancienne du Néolithique final) (Chambon et Salanova, 1996). Les deux éléments lithiques recueillis peuvent appartenir au Néolithique moyen ou au Néolithique récent-final, les armatures tranchantes disparaissant progressivement dans le courant du Néolithique final. Une armature unique et qui plus est fragmentée par le feu peut difficilement être un élément de datation fine.

L'industrie osseuse est beaucoup plus exceptionnelle et mérite un inventaire exhaustif des sources de comparaison, en partie déjà réalisé (Pape, 1982). Le site le plus proche est celui d'Isles-les-Meldeuse (Seine-et-Marne) (Bailloud, 1961), malheureusement découvert dans un contexte incertain (n 15 à 19, fig. 12). S’y juxtaposent en effet de longues pointes à soie, de longs poinçons et des lames sur base de métapode; le site fut attribué à l'époque au SOM.

On retrouve l'association de longs poinçons et de pointes à soie dans la sépulture collective non mégalithique de Stein (Pays-Bas) (n ${ }^{\circ} 20$ et 21, fig. 12) (Moddermann, 1964), dans un contexte du Néolithique final complété par la présence d'une bouteille à collerette. Récemment, la sépulture collective de Vignely (Seine-et-Marne) a livré un ensemble de pointes du même type (fouilles sous la direction de Y. Lanchon : Allart et al., 1998). La datation de la couche sépulcrale y fournit un intervalle comparable à celle de la structure 1 de Poses: Ly-9401 [3517-3357] av. J.-C. en datation calibrée (in Mille et Bouquet, 2004).

À côté de ces deux sites, W. Pape (1982) en a isolé plusieurs autres ayant livré le même type de pointe allongée à soie aplatie à l'extrémité et à feuille sans barbelure ( $\mathrm{n}^{\circ} 1$ à 14 , fig. 12). Ces pointes se répartissent de l'Île-de-France à l'Allemagne du Nord et de la Hollande à l'Allemagne orientale. Elles appartiennent pour la plupart au Néolithique récent-final et proviennent de sépultures collectives du complexe SOM (Montigny-Esbly, Crécy-en-Brie, Oyes, Coizard), d'allées couvertes de Hesse ou de Westphalie, ou d'habitats du groupe de Bernburg (Schrikel, 1976). Elles ont été également découvertes dans la sépulture collective non mégalithique de Schönstedt en Thüringe (Feustel et Ullrich, 1965; Feustel, 1972).

Un autre élément de chronologie (terminus post quem) est fourni par le mobilier provenant de la grande dépression périphérique colmatée par des limons sableux et plus particulièrement de deux concentrations céramiques numérotées 1 et 2 sur la fig. 2 (tessons 1 à 4 , fig. 10). Ce mobilier est constitué pour l'essentiel de céramique orangée à brun orangé, à dégraissant siliceux grossier. Les formes représentées sont des anses plates ou en boudin ainsi qu'un gros bouton placé quelques centimètres sous le bord. Ce type d'anse peut perdurer de la fin du Néolithique final au Bronze ancien-moyen.

La datation du mobilier associé directement aux vestiges funéraires peut donc s'accorder avec une phase de transition entre le Néolithique moyen et le Néolithique récent, tout en insistant sur le caractère original d'un tel assemblage par rapport au mobilier funéraire connu à cette époque (absence de hache et de parures en particulier). Insistons également sur le fait que la datation ${ }^{14} \mathrm{C}$ de la structure 2 peut tout à fait se raccorder à des événements terminaux dans l'histoire du monument. La seconde datation, réalisée sur os brûlé, ne fait alors que renforcer l'hypothèse d'une construction ancienne, plus ancienne en tout cas que le groupe des allées sépulcrales, dont la phase d'édification semble bien se limiter à la fin du $4{ }^{e}$ millénaire dans le Bassin parisien (Chambon et Salanova, 1996).

\section{Relations avec les sites d'habitat voisins}

Si le monument funéraire de Poses semble unique dans ce secteur de la Boucle du Vaudreuil, il est accompagné d'au moins deux sites d'habitat proches et dont la datation au Néolithique récent est bien attestée : Poses, les Quatre Chemins (Billard et Penna, 1995) et Poses, la Fosse Sulpice - zone D (Aubry, 1994; fouille de A. Bogusewski, rapport non disponible). Compte tenu de l'ampleur des travaux de suivi archéologique qu’a connu ce territoire, cette présence est significative d'un lien entre sépulture et habitat qui reste généralement très difficile à mettre en évidence.

\section{L'architecture}

Sur le plan de la forme architecturale, les données sont lacunaires du fait de l'arasement partiel du tumulus empierré. La question de l'accès à la zone sépulcrale (peut-être au nordouest) n'est pas documentée. Des traits généraux se dessinent malgré tout : édifice probablement circulaire utilisant à la fois terre et pierre, à chambre quadrangulaire large, faiblement encaissée.

Le monument de Poses est relativement éloigné des formes connues dans le Bassin parisien, qu'il s'agisse des allées sépulcrales (mégalithiques ou non), des "cabanes funéraires" (Blanchet et al., 1982 et 1993; Masset, 1995) ou autres hypogées. Seuls certains caractères le rapprochent des sépultures collectives voisines de Val-de-Reuil et Portejoie : c'est d'une part la nature enterrée de la zone de dépôt funéraire 
et d'autre part le recours à la craie, matériau étranger au site qu'il a fallu aller extraire à flanc de coteau. Il convient également de souligner la place importante que le bois a pu prendre dans la construction de la partie excavée de cet édifice, comme ce fut le cas pour les sépultures de Val-de-Reuil " les Varennes » et de Portejoie "Sépulture 1 ». La présence de deux sortes d' " antennes » sur le côté sud-est de la partie centrale fait écho au dispositif d'entrée dans les sépultures collectives non mégalithiques de Bazoches-sur-Vesle (Aisne) (Leclerc, 1995; Chambon, 2003) ou bien de Vignely (Seineet-Marne) (Allard et al., 1998), qui s'expliquent par l'utilisation d'une architecture sur sablière basse en bois.

Le principe d'une évolution continue de l'architecture mégalithique, concernant en particulier son caractère monumental et les proportions entre chambre et couloir, est aujourd'hui acquis (L'Helgouac'h, 1973; Boujot et Cassen, 1992). La forme massive et circulaire de l'édifice tumulaire de Poses rappelle certains monuments de la Plaine de Caen (Ernes, Condé-sur-Ifs en particulier) et la forme rectangulaire de sa " chambre " évoque davantage des monuments du Néolithique atlantique à chambre rectangulaire, appartenant aux tombes à couloir à chambre simple tels que les dolmens de type angevin, considérés comme tardifs dans la chronologie du Néolithique moyen. Ces types de monuments, lorsqu'ils n'ont pas été réutilisés au Néolithique final, ne livrent quantitativement que peu de mobilier funéraire. Une datation proche de la fin du Néolithique moyen peut être proposée et on est tenté de voir dans le monument de Poses une forme de transition architecturale entre les tumulus avec chambre à couloir du Néolithique moyen atlantique et les allées sépulcrales enterrées du Néolithique récent.

Parmi les cinq monuments de Val-de-Reuil et Portejoie (Billard et al. [dir.], en préparation), des datations réalisées sur trois d'entre eux permettent de situer la phase de construction de ces allées sépulcrales dans une même période chronologique, soit au début du Néolithique récent, vers la fin du quatrième millénaire avant J.-C. La fourchette commune aux trois datations se place entre 3300 et 3100 avant J.-C. en dates calibrées. La structure 1 de Poses est donc antérieure à la phase d'édification des allées sépulcrales, même si la datation fournie par la structure 2, qui correspond à un événement probablement terminal, permet d'envisager que le fonctionnement de ce monument se prolonge jusqu'à une phase charnière qui voit l'apparition de nouvelles formes architecturales.

Le monument de Poses et la place de l'incinération dans les sépultures collectives néolithiques

Le but de cet article ne vise pas à la recherche exhaustive des monuments présentant une architecture ou des prati- ques funéraires voisines. On peut toutefois insister sur le fait que la pratique de l'incinération est loin d'apparaître comme anecdotique, même si l'on met à part les cas manifestes de sépultures incendiées, assez nombreux aujourd'hui dans le Bassin parisien (cf. Guillot et Le Goff, 1995; Masset, 2002; Gatto, 2003), telles que le « crématoire SOM » de La Hoguette à Fontenay-le-Marmion (Calvados), très probablement une allée sépulcrale en bois incendiée (Caillaud et Lagnel, 1972). Au total, L. Burnez-Lanotte (1987) a répertorié 29 sites funéraires du complexe SOM livrant des restes humains brûlés.

Rappelons également qu'au Néolithique moyen le site de Mestreville à Saint-Pierre-d'Autils (Eure), fouillé anciennement par A. G. Poulain et dont l'étude a été reprise récemment (Billard et al., 1988; Billard, 1990), a montré qu'au moins trois abris sous roche avaient donné lieu à une utilisation funéraire complexe au Néolithique moyen. En particulier, la couche B de l'abri $n^{\circ} 1$ (ou " abri du mammouth ") a livré un foyer contenant une mandibule d'adolescent au milieu d'un amas de pièces lithiques et d'ossements animaux. L'abri n ${ }^{\circ} 2$ (ou " abri du squelette ") a livré trois foyers (dont le dernier fouillé en 1988) comportant des fragments de crânes humains brûlés ainsi qu'une clavicule humaine, des ossements animaux partiellement brûlés (bovidés, cerf, oiseaux), de nombreux silex taillés et des tessons de céramique néolithique. Dans ce dernier cas, cette pratique d'incinération pourrait être liée à une sorte de caveau situé dans le fond de l'abri et ayant livré, lors des fouilles anciennes, un squelette complet (non brûlé).

À Neuvy-en-Dunois (Eure-et-Loir) (Masset et al., 1968), l'une des plus anciennes sépultures collectives du Bassin parisien (datée [4354-3764] avant J.-C. en datation calibrée) ne contenait que des restes d'individus incinérés. Plus récente, la sépulture de Maison Rouge à Montigny (Loiret) est datée du Néolithique récent et abritait les restes de plusieurs dizaines d'individus qui ont été incinérés à l'extérieur du caveau (Masset et Baratin, 1980 : Gif 3759 et 3760, soit respectivement [3346-2507] et [3616-2879] avant J.-C. en datations calibrées). Citons également, parmi les sépultures offrant témoignage d'une pratique de l'incinération, la « cabane funéraire » de Noisy-sur-École (Seine-et-Marne), une autre sépulture collective dont les dimensions et les datations ne sont pas sans évoquer la chambre rectangulaire de Poses (Brézillon et al., 1973). Le monument de Marolles I, interprété comme allée couverte mégalithique, contenait, quant à lui, huit à dix corps incinérés (Masset et Mordant, 1967).

Le caveau non mégalithique de Stein (Modderman, 1964) a livré les restes incinérés d'une trentaine d'individus rassemblés en deux grands tas. Cependant, d'éventuelles inhumations ont pu y disparaître à cause de l'acidité du sol. Encaissé d'environ $50 \mathrm{~cm}$ sous le sol actuel, le monument a livré un 
mobilier funéraire très proche par sa composition de celui de Poses, en particulier une série de pointes en os exposées au feu ainsi qu'un pot à fond plat assez proche de celui de Poses par sa forme et la relative finesse de ses parois. D'autres types de mobilier plus variés qu'à Poses ainsi qu'une datation ${ }^{14} \mathrm{C}$ tardive (vers 2600 avant J.-C. - calibré) distinguent toutefois les deux monuments.

La pratique de l'incinération est également attestée dans le Steinkist de Lohra en Hesse ou bien dans la sépulture collective de Derenburg. Incinération et inhumation coexistent par exemple à Hérouval (Montjavoult I, Val-d'Oise), à Gudensberg en Hesse, à Nennungen en Saxe. Quelques exemples d'incinération figurent en Grande-Bretagne et en Irlande (culture de Clyde-Carlingford, long barrows de la culture de Windmill-Hill, cultures de la Boyne et de Dorchester). Dans le Bassin parisien, il est de plus courant de rencontrer quelques ossements brûlés parmi les restes inhumés. Au total, de nombreux cas de figure existent et laissent présager que le feu a pu avoir des fonctions multiples au sein des caveaux collectifs néolithiques.

À Poses, il ne semble pas que le traitement par le feu ait été appliqué à l'édifice mais bien aux restes des défunts et à une partie du mobilier funéraire. Par ailleurs, le lieu a pu fonctionner comme un ossuaire, recevant les restes des défunts en une seule fois. S'ajoute à cette pratique funéraire un type d'architecture incontestablement originale qui pourrait renvoyer au mégalithisme atlantique et annoncer une évolution vers les sépultures collectives dissimulées du Bassin parisien. À l'intérieur de la microrégion que forme la confluence de la Seine et de l'Eure, on distingue toutefois des allées sépulcrales (mégalithiques ou non), édifiées à une date très proche mais postérieure à celle obtenue pour le monument de Poses. Dans ce dernier, nous n'avons rencontré ni l'ampleur des dépôts funéraires de ces allées sépulcrales, ni une longévité qui serait attestée par la multiplicité et la variété du mobilier. Surtout, aucune des cinq sépultures collectives de Val-de-Reuil et Portejoie n’a livré de témoins d'une incinération quelconque des ossements.

Cette originalité s'explique très probablement par la position chronologique de l'édifice de Poses. Au sein de la microrégion que constitue la Boucle du Vaudreuil, on ne peut manquer également de remarquer la présence d'un outillage osseux particulièrement soigné, dont il n'existe aucun équivalent dans les allées sépulcrales environnantes. De plus, le monument de Poses semble isolé alors que ces allées sépulcrales sont regroupées dans un ensemble. Cette différence de traitement des individus ne nous semble pas anecdotique et pourrait être significative soit de différences de statuts individuels au sein de la société néolithique, soit des mutations qui affectent le domaine funéraire entre le Néolithique moyen et le Néolithique récent.

\section{Conclusions}

À Poses, seules des conditions exceptionnelles ont permis la conservation d'une partie de la superstructure de l'édifice. Dans d'autres conditions moins bonnes, on peut imaginer que les vestiges correspondant à des sépultures $\mathrm{du}$ même type et dont le caractère monumental est indéniable se réduisent à une fosse peu profonde dans laquelle figurent quelques esquilles d'ossements brûlés et quelques tessons. La difficulté à identifier ce type de vestiges pourrait expliquer le manque de documentation concernant les pratiques funéraires immédiatement antérieures à la génération des allées sépulcrales dans le Bassin parisien.

Le site de Poses soulève donc plus globalement la question de l'identité funéraire des groupes néolithiques du Bassin parisien face au mégalithisme atlantique, lorsque l'on considère la période comprise entre le premier monumentalisme (Sépultures de type Passy, longs tumulus) et l'apparition des allées sépulcrales. Il est difficile de croire que l'on y a délaissé toute construction monumentale. Peut-on concevoir alors qu'au sein du Bassin parisien se soient développées au Néolithique moyen des architectures funéraires originales, voisines par la forme architecturale à défaut de l'être par les matériaux utilisés? Nous l'avons dit, ce monument doit son identification à des conditions exceptionnelles qui ne sont que rarement réunies dans les zones de grandes cultures. C'est pourquoi nous pensons qu'au stade actuel de la recherche dans le nord de la France, il serait regrettable d'opposer deux zones géographiques, l'une atlantique où l'attention s'est focalisée sur l'architecture mégalithique, et l'autre, plus à l'est, où la forte activité de l'archéologie préventive tend à mettre en avant les sépultures en fosse.

\section{Bibliographie}

Allard, P., André, M.-F., Chambon, P., Lafage, F. et Praud, Y., 1998 - La sépulture collective de Vignely, La Porte aux Bergers (Seine-et-Marne), Le Néolithique du Centre-Ouest de la France, (actes du XXI ${ }^{e}$ colloque interrégional sur le Néolithique - Poitiers 1994), Chauvigny, Association des Publications Chauvinoises, p. 395-401.

Aubry, B., 1994 - Poses "Sur la Mare " et "La Fosse Sulpice ", (Rapport de diagnostic archéologique), Rouen, Service régional de l'Archéologie de Haute-Normandie.

Bailloud, G., 1961 - Un remarquable ensemble SOM provenant d'Isles-les-Meldeuses (Seine-et-Marne), Bulletin de la Société préhistorique française, 68, p. 398-406.

Baudot, M.,1944 - Premier rapport sur la fouille du caveau sépulcral de Pinterville (Eure), Bulletin de la Société normande d'Études préhistoriques et historiques, 34-1, p. 4-7. 
Baudot, M. et Gaudron, G., 1943 - Rapport sur les fouilles de l'allée sépulcrale de Pinterville (Eure) (document dactylographié), Rouen, Service régional de l'Archéologie de HauteNormandie, 5 p.

Billard, C., 1990 - L'étude des pratiques funéraires chasséennes au travers des fouilles des abris-sous-roche de Mestreville à Saint-Pierre-d'Autils (fouilles anciennes et récentes), in G. Fosse et A. Ropars (dir.), L’Eure de la Préhistoire (catalogue d'exposition), Musée de Vernon, p. 31-37.

Billard, C., Chambon, P. et Guillon, M., 1995 - L'ensemble des sépultures collectives de Val-de-Reuil et Portejoie (Eure) : présentation, (Actes du $\mathrm{XX}^{\mathrm{e}}$ colloque interrégional sur le Néolithique - Amiens 1992), Revue archéologique de Picardie ( ${ }^{\circ}$ spécial), 9, p.147-154.

Billard, C., Fosse, G., Lebret, P. et Ropars, A., 1988 - Les abris-sous-roche de Mestreville (commune de Saint-Pierre-d'Autils - Eure), (DFS), Rouen, Service régional de l'Archéologie de Haute-Normandie, 21 p., 18 fig., annexes.

Billard, C., Guillon, M. et Verron, G. (dir.), en préparation - Les sépultures collectives de Val-de-Reuil et Portejoie (Eure).

Billard, C. et Penna, B., 1995 - Les sites de Poses « Les Quatre Chemins" et "La Plaine de Poses" (Eure) : transition Néolithique moyen-récent et Campaniforme. Revue archéologique de l'Ouest, suppl. $\mathrm{n}^{\circ} 7$ (actes du $20^{\mathrm{e}}$ colloque interrégional sur le Néolithique - Évreux 1993), p.273-291.

Blaizot, F. et Bö̈s, X., 2003 - Une crémation primaire multiple en fosse au néolithique final: la tombe-bûcher de Reichstett-Mundolsheim-Souffelsweyersheim (Bas-Rhin), in E. Derwich (dir.), Préhistoire des pratiques mortuaires, Liège, Eraul, p. 49-60.

Blanchet, J.-C., Delsaux, M.-A., Huysecom, E. et Woimant, G.-P., 1982 - La cabane funéraire collective SOM du Hazoy à Compiègne (Oise), Bulletin de la Société préhistorique française, 79-8, p. 227.

Blanchet, J.-C., Delsaux, M.-A., Huysecom, E. et Woimant, G.-P., 1993 - La cabane funéraire SOM de Compiègne « Le Hazoy " (Oise), Revue archéologique de Picardie, 3/4, p. 4170.

Bonnin, T., 1843 - Notice sur un tombeau celtique découvert en décembre 1842 à Saint-Étienne du Vauvray (Eure), Évreux, Ancelle, 15 p.

Bostyn, F. (dir.), Beurion, C., Billard, C., Guillon, M., Hachem, L., Hamon, C., Lanhon, Y., Praud, Y., Reckinger, F., Ropars, A. et Munaut A.-V., 2003 - Néolithique ancien en Haute-Normandie : le village Villeneuve-Saint-Germain de Poses «Sur la Mare » et les sites de la Boucle du Vaudreuil, Paris, Société préhistorique française, coll. "Travaux; 4 », 343 p.

Boujot, C. et Cassen, S., 1992 - Le développement des premières architectures funéraires monumentales en France occidentale, in C.-T. Le Roux (dir.), Paysans et bâtisseurs, l'émergence du Néolithique atlantique et les origines du mégalithisme. (actes du $17^{\mathrm{e}}$ colloque interrégional sur le Néolithique - Vannes 1990). Revue archéologique de l'Ouest, Suppl. n 5, p. 195 211.

Brézillon, M., Degros, J., Girard, C., Girard, M. et TArrête, J., 1973 - La sépulture collective du Paradis à Noisy-sur-École (Seine-et-Marne), Cahiers du Centre de recherches protohistoriques, université de Paris 1, 2, p. 4-75.

Burnez-Lanotte, L., 1987 - Le Chalcolithique moyen entre la Seine et le Rhin inférieur, Oxford, British Archaeological Reports (International Series, 354), 3 vol.

Buikstra, J. E. et Swegle, M., 1989 - Bone modification due to burning : experimental evidence, in R. BonNichsen et M. H. Sorg (ed.), Bone modification, Center for the study of the first americans, p. 247-258.

Caillaud, R. et Lagnel, E., 1972 - Le cairn et le crématoire de la Hoguette à Fontenay-le-Marmion (Calvados), Gallia Préhistoire, 15-1, p. 137-185.

Снамвon, P., 1995 - L'ossuaire du Néolithique récent à Berry-auBac (Aisne) : une structure post-funéraire?, Revue archéologique de Picardie, 1/2, p. 61-81.

Снамвоn, P., 2003 - Les morts dans les sépultures collectives néolithiques en France. Du cadavre aux restes ultimes. (35e supplément à Gallia Préhistoire), Paris, CNRS, 395 p.

Chambon, P. et Salanova, L., 1996 - Chronologie des sépultures du III ${ }^{\mathrm{e}}$ millénaire dans le bassin de la Seine, Bulletin de la Société préhistorique française, 93-1, p. 103-115.

Clet-Pellerin, M., 1986 - Analyses polliniques dans quelques sites néolithiques de Normandie. (actes du $\mathrm{X}^{\mathrm{e}}$ colloque interrégional sur le Néolithique - Caen 1983), Revue archéologique de l'Ouest, suppl. $\mathrm{n}^{\circ} 1$, p. 279-284.

Collignon, M., 1928-1930 - Le déplacement du menhir de la Basse-Crémonville, près de Louviers, en 1866, Bulletin de la Société d'Études diverses de l'Arrondissement de Louviers, 20, p. 30-38.

Coutil, L., 1896 - Inventaire des menhirs et dolmens de France, Eure, Bulletin de la Société normande d'Études préhistoriques et historiques, 4, p. 57-62.

Divry, A., 1944 - L'allée sépulcrale néolithique de Pinterville. Bulletin de la Société normande d'Études préhistoriques et historiques, 34-2, p. 24-25.

DudaY, H., 1987 - La quantification des restes humains. Application à l'étude des sépultures à incinération, ou des différentiels autres que la conservation, Actes de la table-ronde de la RCP 742 du CNRS (Saint-Germain-en-Laye, mai 1987), p. 17-22.

Feustel, R. et Ullrich, H., 1965 - Totenhütten der neolithischen Waltienburger Gruppe - Alt-Thüringen, 7, p. 105-202, $27 \mathrm{pl}$.

Feustel, R., 1972 - Die Walternienburge/Bernburger Totenhütte von Schönstedt im Thüringer Becken, Alt-Thüringen, 12, p. 31-114. 
Gatto, E. 2003 - La place de la crémation dans le traitement des défunts à la fin du Néolithique en France, Thèse d'Anthropologie biologique, Université de Bordeaux 1.

Guillot, H. et Le Goff, I., 1995 - Les usages du feu dans les sépultures collectives du Bassin parisien, in C. Masset, et P. Soulier, (dir.), Allées couvertes et autres monuments funéraires du Néolithique dans la France du nord-ouest, Paris, Errance, p. 112-113.

Haldimann, M.-A., Ramjoue, E. et Simon, C., 1991 - Les fouilles de la cour de l'ancienne prison de St-Antoine: une vision renouvelée de la Genève antique, Archéologie Suisse, 14, p. 194-204.

Hamy, E. T., 1874 - Sur les ossements du dolmen des Vignettes à Léry. Bulletin de la Société d'Anthropologie de Paris, 9, p. 606-608.

Krogman, W. M. et Iscan, M. Y., 1986 - The Human Skeleton in Forensic Medicine, Springfield (Illinois), Charles C. Thomas publisher [2édition révisée de Krogman, 1962].

Langlois, J.-Y. et Celly, P., 1995 - Poses - Sur la Mare, (DFS), Rouen, Service régional de l'Archéologie de HauteNormandie.

Lanting, J. et Brindley, A, 2005 - La datation des os calcinés, in J. Bourgeois, et M. TAlon, L'áge du Bronze du nord de la France dans son contexte européen, Paris, CTHS, p. 35-42.

LeClerc, J., 1995 - Bazoches-sur-Vesle (Aisne), in C. Masset et P. Soulier, (dir.), Allées couvertes et autres monuments funéraires du Néolithique dans la France du nord-ouest, Paris, Errance, p. 137-138.

LE GoFf, I., 1998 - De l'os incinéré aux gestes funéraires. Essai de paléoethnologie à partir des vestiges de la crémation, (thèse de Préhistoire, ethnologie, anthropologie), Université de Paris I (2 vol.), 945 p.

Le Goff, I., Billand, G. et Guillot, H., 2002 - Histoire d'une sépulture collective néolithique incendiée à Lacroix-SaintOuen, in M. A. Rojo Guerra et M. Kunst (dir.), Sobre el significado del fuego en los rituales funerarios del neolitico, Valladolid, Universidad de Valladoid (Studia Archaeologica, 91), p. 127-146.

L'Helgouac'H, J., 1973 - Les mégalithes de l'ouest de la France : évolution et chronologie, (actes du III $^{\mathrm{e}}$ colloque atlantique - Moesgard 1969), Köbenhavn, Jutland Archaelogical Society Publications (11), p. 203-219.

Maire, J.-L., 1983 - La nécropole d'Epiais-Rhus (Val-d'Oise), approche du rituel funéraire gaulois à travers les déformations des céramiques (urnes à incinération). Revue archéologique de Picardie, 1, p. 159.

Marquer, P. , 1954 - Les ossements humains de Pinterville (Eure), Bulletin et Mémoires de la Société d'Anthropologie de Paris, 5 (36), p. 209-235.

Masset, C., 1995 - Cabanes funéraires, Bulletin de la Société préhistorique française, 92-1, p. 107-108.

Masset, C. 2002 - Ce que l'on sait, ou croit savoir, du rôle du feu dans les sépultures collectives néolithiques, in M. A. Rojo
Guerra et M. Kunst (dir.), Sobre el significado del fuego en los rituales funerarios del neolitico, Valladolid, Universidad de Valladoid (Studia Archaeologica, 91), p. 9-20.

Masset, C., Demetz, J.-L., Baron, R. et Girard, M., 1968 - Les incinérations du Néolithique ancien de Neuvy-en-Dunois, Gallia Préhistoire, 11, p. 205-234.

Masset, C. et Mordant, D. et C., 1967 - Les sépultures collectives de Marolles-sur-Seine (Seine-et-Marne). Gallia Préhistoire, 10, p. 75-136.

Masset, C. et Baratin, J.-F., 1980 - La sépulture à incinérations de la Maison Rouge à Montigny (Loiret), Études sur le Néolithique de la région Centre, (actes du $5^{\mathrm{e}}$ colloque interrégional sur le Néolithique - Saint-Amand-Montrond 1977), Saint-Amand-Montrond, Association des Amis du Musée Saint-Vic, p. 141-147.

Mille, B. et Bouquet, L., 2004 - Le métal au $3^{\mathrm{e}}$ millénaire dans le Centre-Nord de la France, in M. Van der Linden et L. Salanova (dir.), Le $3^{\text {e }}$ millénaire dans le Nord de la France et en Belgique. Anthropologica et Praehistorica, 115, p. 197-215.

ModdermanN, P. J .R., 1964 - The Neolithic Burial Vault at Stein. Analecta Prehistorica Leidensia, I, p. 3-16.

PaPe, W., 1982 - Au sujet de quelques pointes de flèche en os. Industrie de l'os néolithique et de l'âge des métaux 2, Paris, CNRS, p. 135-171.

Person, A., Saliège, J.-F., Gérard, M. et Paris, F., 1998 Utilisation d'un indice caractéristique de la diagenèse de la fraction minérale d'ossements archéologiques en milieu désertique pour discuter de la fiabilité de ces matériaux comme support de datation par le radiocarbone, application à deux nécropoles néolithiques de l'Aïr (Niger), Études nigeriennes, p. 77-78.

Saliège, J.-F., Person, A. et Paris, F., 1998 - Datation du carbonate-hydroxylapatite d'ossements holocènes du Sahel (Mali, Mauritanie, Niger), (pré-actes du $3^{e}$ colloque international ${ }^{14} \mathrm{C}$ et archéologie - Lyon 1998), p. 172-173.

Schrikel, W., 1976 - Die Galeriegrabkultur Westdeutschlands, Die Anfänge des Neolithikums vom Orient bis Nordeuropa, Köln/Wien, Böhlau Verlag (Fundamenta A/3, Teil Vb), p. 188-239.

TineveZ, J.-Y., 1988 - La sépulture à entrée latérale de Beaumont en Saint-Laurent-sur-Oust, Revue archéologique de l'Ouest., 5, p. 55-78.

Tinevez, J.-Y., Baud C.-A., Grevin G., Lagier R., Giot, P.-R. et Morzadec, H., 1990 - La sépulture mégalithique à entrée latérale de Beaumont à Saint-Laurent-sur-Oust (Morbihan) : études anthropologique et pétrographique; données complémentaires, Revue archéologique de l'Ouest, 7, p 41-56.

UbelaKer, D. H., 1978 - Human Skeletal Remains. Excavation, Analysis, Interpretation, Chicago, Taraxacum, Aldine Publishing Co., $118 \mathrm{p}$.

Verron, G., 1975 - Les sépultures collectives néolithiques de Portejoie (Eure), Nouvelles de l'Eure, 56, p. 49-54. 


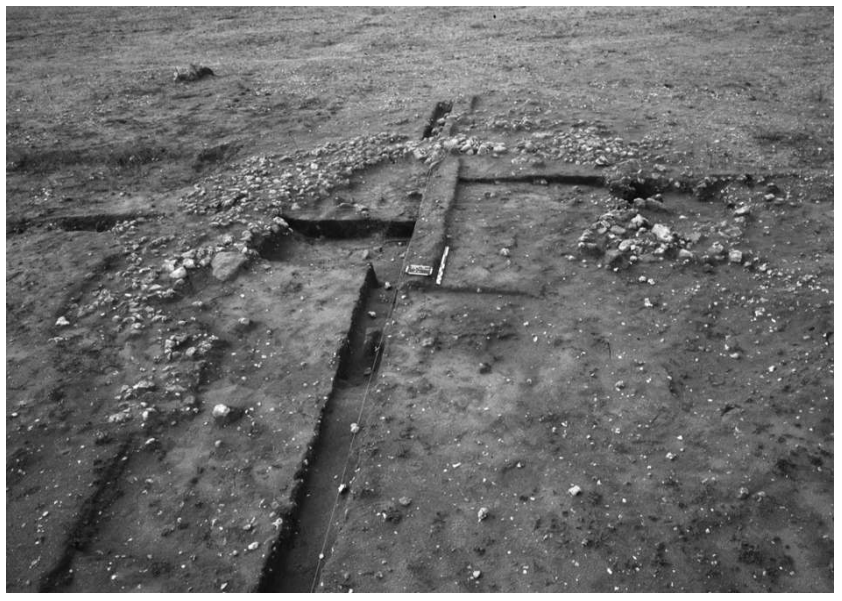

Photo 1 : Vue d'ensemble de l'empierrement en cours de fouille. Photo 1: General view of the stone surround in course of excavation.

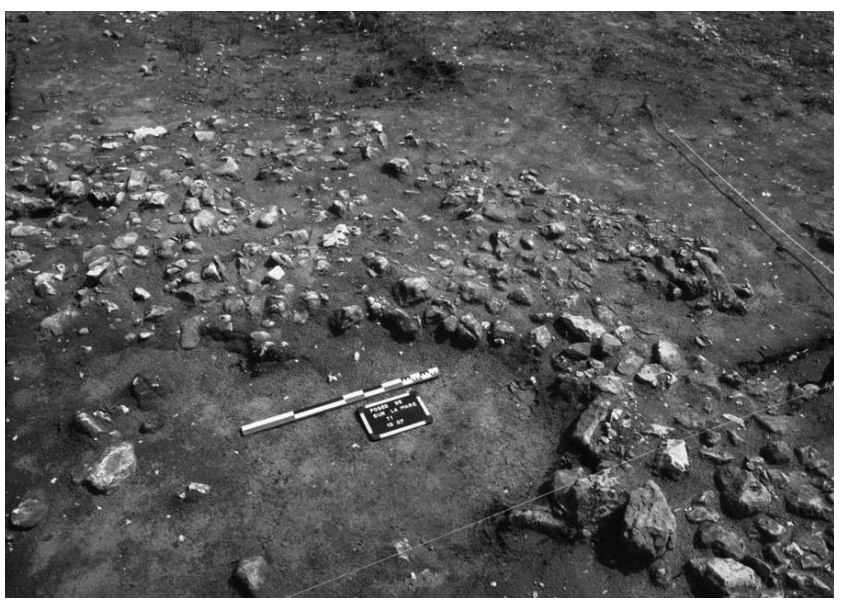

Photo 3 : Détail de la partie sud de l'empierrement. Photo 3: Detail of the southern part of the stone surround.

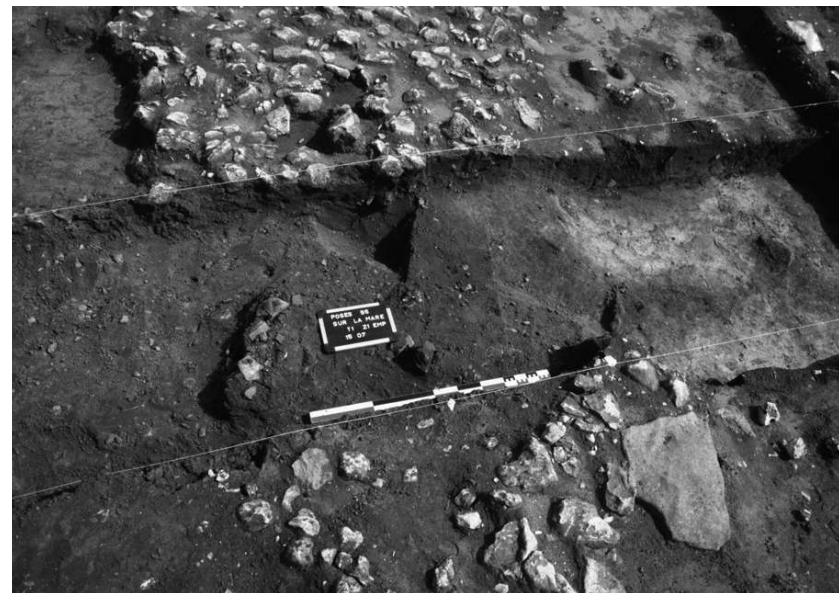

Photo 2 : Coupe dans l'empierrement au niveau de la zone 1. Photo 2: Section of the stone surround in zone 1.

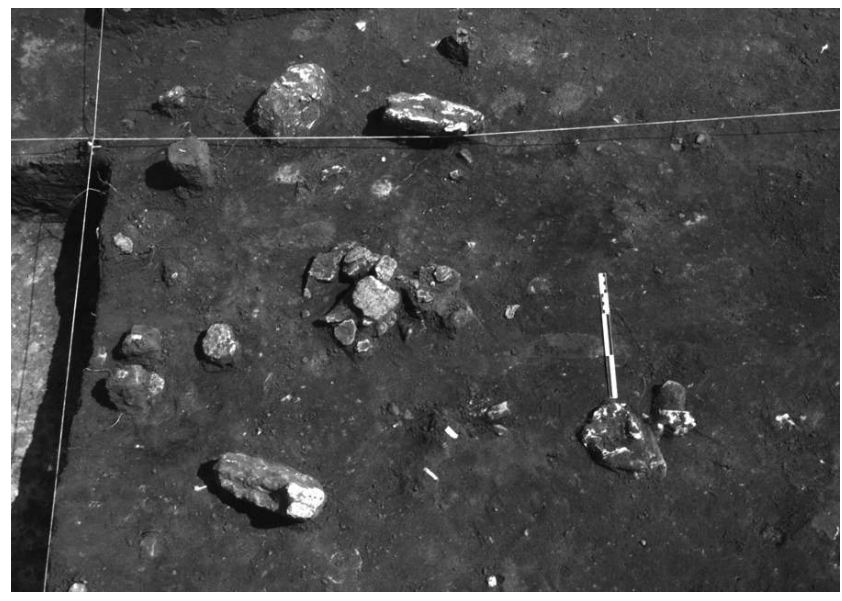

Photo 4 : Vue d'ensemble de la structure 2 en cours de fouille (le Nord est à gauche).

Photo 4: General view of feature 2 during excavation (North is on the left).

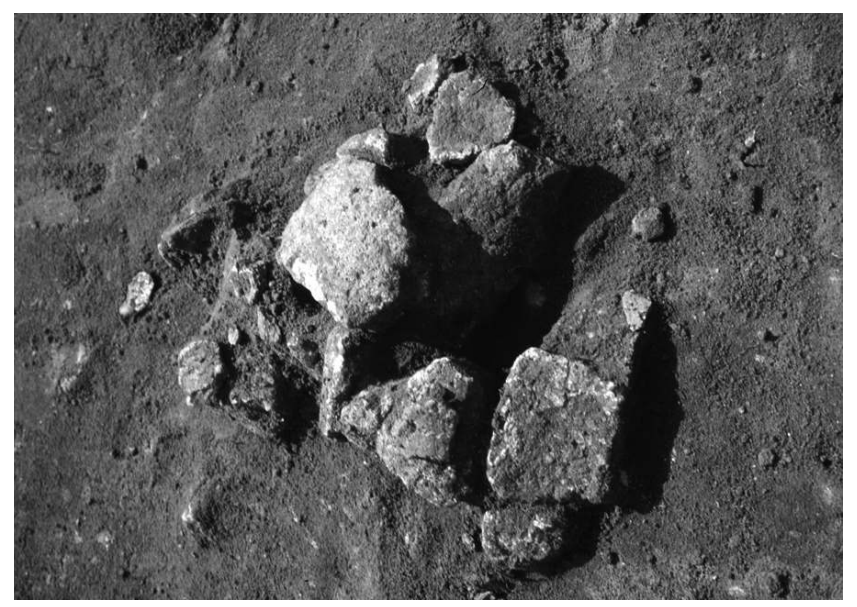

Photo 5 : Amas de tessons du vase de la structure 2.

Photo 5: Shard concentration in feature 2. 